\title{
THE WALDSCHMIDT CONSTANT FOR SQUAREFREE MONOMIAL IDEALS
}

\author{
CRISTIANO BOCCI, SUSAN COOPER, ELENA GUARDO, BRIAN HARBOURNE, MIKE \\ JANSSEN, UWE NAGEL, ALEXANDRA SECELEANU, ADAM VAN TUYL, AND THANH VU
}

\begin{abstract}
Given a squarefree monomial ideal $I \subseteq R=k\left[x_{1}, \ldots, x_{n}\right]$, we show that $\widehat{\alpha}(I)$, the Waldschmidt constant of $I$, can be expressed as the optimal solution to a linear program constructed from the primary decomposition of $I$. By applying results from fractional graph theory, we can then express $\widehat{\alpha}(I)$ in terms of the fractional chromatic number of a hypergraph also constructed from the primary decomposition of $I$. Moreover, expressing $\widehat{\alpha}(I)$ as the solution to a linear program enables us to prove a Chudnovsky-like lower bound on $\widehat{\alpha}(I)$, thus verifying a conjecture of Cooper-EmbreeHà-Hoefel for monomial ideals in the squarefree case. As an application, we compute the Waldschmidt constant and the resurgence for some families of squarefree monomial ideals. For example, we determine both constants for unions of general linear subspaces of $\mathbb{P}^{n}$ with few components compared to $n$, and we find the Waldschmidt constant for the Stanley-Reisner ideal of a uniform matroid.
\end{abstract}

\section{INTRODUCTION}

During the last decade, there has been a lot of interest in the "ideal containment problem": given a nontrivial homogeneous ideal $I$ of a polynomial ring $R=k\left[x_{1}, \ldots, x_{n}\right]$ over a field $k$, the problem is to determine all positive integer pairs $(m, r)$ such that $I^{(m)} \subseteq I^{r}$. Here $I^{(m)}$ denotes the $m$-th symbolic power of the ideal, while $I^{r}$ is the ordinary $r$-th power of $I$ (formal definitions are postponed until the next section). This problem was motivated by the fundamental results of [10, 20] showing that containment holds whenever $m \geq r(n-1)$. In order to capture more precise information about these containments, Bocci and Harbourne [3] introduced the resurgence of $I$, denoted $\rho(I)$ and defined as $\rho(I)=\sup \left\{m / r \mid I^{(m)} \nsubseteq I^{r}\right\}$.

In general, computing $\rho(I)$ is quite difficult. Starting with [3], there has been an ongoing research programme to bound $\rho(I)$ in terms of other invariants of $I$ that may be easier to compute. One such bound is in terms of the Waldschmidt constant of $I$. Given any nonzero homogeneous ideal $I$ of $R$, we let $\alpha(I)=\min \left\{d \mid I_{d} \neq 0\right\}$; i.e., $\alpha(I)$ is the smallest degree of a nonzero element in $I$. The Waldschmidt constant of $I$ is then defined to be

$$
\widehat{\alpha}(I)=\lim _{m \rightarrow \infty} \frac{\alpha\left(I^{(m)}\right)}{m} .
$$

2010 Mathematics Subject Classification. Primary 13F20; Secondary 13A02, 14N05.

Key words and phrases. Waldschmidt constant, monomial ideals, symbolic powers, graphs, hypergraphs, fractional chromatic number, linear programming, resurgence.

Last updated: Revised Version (May 21, 2016). 
This limit exists and was first defined by Waldschmidt 28] for ideals of finite point sets in the context of complex analysis. In the language of projective varieties, Waldschmidt was interested in determining the minimal degree of a hypersurface that passed through a collection of points with prescribed multiplicities, that is, he was interested in determining $\alpha\left(I^{(m)}\right)$ when $I$ defined a set of points. Over the years, $\widehat{\alpha}(I)$ has appeared in many guises in different areas of mathematics, e.g., in number theory [5, 28, 29], complex analysis [26], algebraic geometry [3, 4, 11, 24] and commutative algebra [18].

Bocci and Harbourne's result $\alpha(I) / \widehat{\alpha}(I) \leq \rho(I)$ (see [3, Theorem 1.2]) has renewed interest in computing $\widehat{\alpha}(I)$. For example, Dumnicki [7] finds lower bounds for $\widehat{\alpha}(I)$ when $I$ is an ideal of generic points in $\mathbb{P}^{3}$, Dumnicki, et al. [8] compute $\widehat{\alpha}(I)$ when $I$ defines a set of points coming from a hyperplane arrangement, Fatabbi, et al. [12] computed $\widehat{\alpha}(I)$ when $I$ defines a special union of linear varieties called inclics, M. Baczyńska, et al. [1] examine $\widehat{\alpha}(I)$ when $I$ is a bihomogeneous ideal defining a finite sets of points in $\mathbb{P}^{1} \times \mathbb{P}^{1}$ in [1]. Guardo, et al. [17] also computed $\widehat{\alpha}(I)$ when $I$ is the ideal of general sets of points in $\mathbb{P}^{1} \times \mathbb{P}^{1}$. In addition, upper bounds on $\widehat{\alpha}(I)$ were studied in [9, 16], along with connections to Nagata's conjecture. Even though computing $\widehat{\alpha}(I)$ may be easier than computing $\rho(I)$, in general, computing the Waldschmidt constant remains a difficult problem.

In this paper we focus on the computation of $\widehat{\alpha}(I)$ when $I$ is a squarefree monomial ideal. After reviewing the necessary background in Section 2, in Section 3 we turn to our main insight: that $\widehat{\alpha}(I)$ can be realized as the value of the optimal solution of a linear program (see Theorem 3.2). To set up the required linear program, we only need to know the minimal primary decomposition of the squarefree monomial ideal $I$. The Waldschmidt constant of monomial ideals (not just squarefree) was first studied in [6] (although some special cases can be found in [2, 14]) which formulates the computation of $\widehat{\alpha}(I)$ as a minimal value problem on a polyhedron constructed from the generators of $I$. Our contribution gives a more effective approach using the well-known simplex method for computing the Waldschmidt constant (see Remark 3.3 for connections to [6]).

The ability to express $\widehat{\alpha}(I)$ as a solution to a linear program has a number of advantages. First, in Section 4 we relate $\widehat{\alpha}(I)$ to a combinatorial invariant. Specifically, we can view a squarefree monomial ideal $I$ as the edge ideal of a hypergraph $H=(V, E)$ where $V=\left\{x_{1}, \ldots, x_{n}\right\}$ are the vertices and $\left\{x_{j_{1}}, \ldots, x_{j_{t}}\right\}$ is an edge (i.e., $\left\{x_{j_{1}}, \ldots, x_{j_{t}}\right\} \in E$ ) if and only if $x_{j_{1}} \cdots x_{j_{t}}$ is a minimal monomial generator of $I$. We then have the following result.

Theorem 1.1 (Theorem 4.6). Suppose that $H=(V, E)$ is a hypergraph with a non-trivial edge, and let $I=I(H)$. Then

$$
\widehat{\alpha}(I)=\frac{\chi^{*}(H)}{\chi^{*}(H)-1} .
$$

where $\chi^{*}(H)$ is the fractional chromatic number of the hypergraph $H$.

Because the fractional chromatic number of a (hyper)graph is a well-studied object (e.g., see the book [23]), Theorem 1.1 enables us to utilize a number of known graph theoretic results to compute some new values of $\widehat{\alpha}(I)$. For example, in Section 6 we compute $\widehat{\alpha}(I)$ when $I$ is an edge ideal for various well-known families of graphs (e.g., bipartite, perfect, cycles). We also show how to simplify the proof of the main result of [2, 14]. Moreover, we 
establish that the Waldschmidt constant of the edge ideal of a graph admits a lower and an upper bound in terms of the chromatic number and the clique number of the graph, respectively.

Second, the reformulation of $\widehat{\alpha}(I)$ as a linear program gives us a new proof technique that allows us to prove a Chudnovsky-like lower bound on $\widehat{\alpha}(I)$ in Section 5. Chudnovsky [5] originally proposed a conjecture on $\widehat{\alpha}(I)$ in terms of $\alpha(I)$ and $n$ when $I$ defined a set of points in $\mathbb{P}^{n}$. Cooper, et al. [6] proposed a Chudnovsky-like lower bound for all monomial ideals. We verify this conjecture in the squarefree case:

Theorem 1.2 (Theorem5.3). Let I be a squarefree monomial ideal with big-height $(I)=e$. Then

$$
\widehat{\alpha}(I) \geq \frac{\alpha(I)+e-1}{e}
$$

We give an example to show that this lower bound is sometimes sharp.

In Section 7, we illustrate how our new technique leads to new containment results, thus returning to the initial motivation for studying Waldschmidt constants. In particular, in this section we study unions of a small number of general linear varieties, the StanleyReisner ideal of a uniform matroid, and a "monomial star", a squarefree monomial ideal of mixed height.

Although we have only focused on squarefree monomial ideals in this paper, our work has implications for the ideal containment problem for a much larger class of ideals. In particular, recent work of Geramita, et al. [15 has shown, among other things, that if $\tilde{I}$ is a specialization of a monomial ideal $I$, i.e. $\tilde{I}$ is obtained by replacing each variable by a homogeneous polynomial with the property that these polynomials form a regular sequence, then $\widehat{\alpha}(\tilde{I})$ and/or $\rho(\tilde{I})$ can be related to $\widehat{\alpha}(I)$ and/or $\rho(I)$ of the monomial ideal (see, for example, [15, Corollary 4.3]).

Acknowledgements. This project was started at the Mathematisches Forschungsinstitut Oberwolfach (MFO) as part of the mini-workshop "Ideals of Linear Subspaces, Their Symbolic Powers and Waring Problems" organized by C. Bocci, E. Carlini, E. Guardo, and B. Harbourne. All the authors thank the MFO for providing a stimulating environment. Bocci acknowledges the financial support provided by GNSAGA of Indam. Guardo acknowledges the financial support provided by PRIN 2011. Harbourne was partially supported by NSA grant number H98230-13-1-0213. Janssen was partially supported by Dordt College. Janssen and Seceleanu received support from MFO's NSF grant DMS-1049268, "NSF Junior Oberwolfach Fellows". Nagel was partially supported by the Simons Foundation under grant No. 317096. Van Tuyl acknowledges the financial support provided by NSERC.

\section{Background Definitions And Results}

In this section we review the relevant background. Unless otherwise indicated, $R=$ $k\left[x_{1}, \ldots, x_{n}\right]$ with $k$ an algebraically closed field of any characteristic. We continue to use the notation and definitions of the introduction. 
2.1. Squarefree monomial ideals and (hyper)graphs. An ideal $I \subseteq R$ is a monomial ideal if $I$ is generated by monomials. We say that $I$ is a squarefree monomial ideal if it is generated by squarefree monomials, i.e., every generator has the form $x_{1}^{a_{1}} \cdots x_{n}^{a_{n}}$ with $a_{i} \in\{0,1\}$. When $I$ is a squarefree monomial ideal, the minimal primary decomposition of $I$ has the form

$$
I=P_{1} \cap \cdots \cap P_{s} \text { with } P_{i}=\left\langle x_{j_{1}}, \ldots, x_{j_{j}}\right\rangle \text { for } j=1, \ldots, s .
$$

A hypergraph is an ordered pair $H=(V, E)$ where $V=\left\{x_{1}, \ldots, x_{n}\right\}$ is the set of vertices, and $E$ consists of subsets of $V$ such that if $e_{i} \subseteq e_{j}$, then $e_{i}=e_{j}$. The elements of $E$ are called edges. When the hypergraph $H$ is such that $\left|e_{i}\right|=2$ for all $i$, it is also called a graph.

Given any hypergraph $H=(V, E)$, we can associate to $H$ a squarefree monomial ideal $I(H)$ called the edge ideal of $H$. Precisely,

$$
I(H)=\left\langle x_{i_{1}} x_{i_{2}} \cdots x_{i_{t}} \mid\left\{x_{i_{1}}, x_{i_{2}}, \ldots, x_{i_{t}}\right\} \in E\right\rangle .
$$

This construction can be reversed, so we have a one-to-one correspondence between hypergraphs $H$ on $n$ vertices and squarefree monomial ideals in $n$ variables.

Remark 2.1. In the above one-to-one correspondence, we need to be cognizant of the fact that a hypergraph with no edges is different than a hypergraph whose edges are the isolated vertices. In the first case, $H=(V, \emptyset)$ is associated to the zero-ideal $I(H)=(0)$, while in the second case, $H=\left(V,\left\{\left\{x_{1}\right\}, \ldots,\left\{x_{n}\right\}\right\}\right)$ is associated to $I(H)=\left\langle x_{1}, \ldots, x_{n}\right\rangle$. In the first case, $\widehat{\alpha}((0))$ is not defined, while in the second case, $\widehat{\alpha}(I(H))=1$ since $I(H)$ is generated by a regular sequence. Thus, it is harmless to eliminate these cases by considering only hypergraphs that have at least one non-trivial edge.

The associated primes of $I(H)$ are related to the maximal independent sets and vertex covers of the hypergraph $H$. We say that $A \subseteq V$ is an independent set of $H$ if $e \nsubseteq A$ whenever $e \in E$. It is maximal if it is maximal with respect to inclusion. A subset $U \subseteq V$ is a vertex cover of a hypergraph if $e \cap U \neq \varnothing$ whenever $e \in E$. A vertex cover is minimal if it is so with respect to containment.

Lemma 2.2. Suppose that $H=(V, E)$ is a hypergraph with a non-trivial edge, and let $I=I(H)$. Suppose that $I=P_{1} \cap \cdots \cap P_{s}$ is the minimal primary decomposition of $I$, and set $W_{i}=\left\{x_{j} \mid x_{j} \notin P_{i}\right\}$ for $i=1, \ldots, s$. Then $W_{1}, \ldots, W_{s}$ are the maximal independent sets of $H$.

Proof. Any $W$ is a maximal independent set if and only if $V \backslash W$ is a minimal vertex cover. We now use the fact that the associated primes of the edge ideal $I(H)$ correspond to the minimal vertex covers of $H$ (e.g., see the proof [27, Corollary 3.35] for edge ideals of graphs, which can be easily adapted to hypergraphs).

2.2. Symbolic Powers. We now review the definition of symbolic powers. Recall that any homogeneous ideal $I \subseteq R$ has minimal primary decomposition $I=Q_{1} \cap \cdots \cap Q_{s}$ where $\sqrt{Q_{i}}=P_{i}$ is a prime ideal. The set of associated primes of $I$, denoted $\operatorname{Ass}(I)$, is the set $\operatorname{Ass}(I)=\left\{\sqrt{Q_{i}} \mid i=1, \ldots, s\right\}$. The minimal primes of $I$, denoted $\operatorname{Min}(I)$, is the set of minimal elements of $\operatorname{Ass}(I)$, ordered by inclusion. 
Definition 2.3. Let $0 \neq I \subseteq R$ be a homogeneous ideal. The $m$-th symbolic power of $I$, denoted $I^{(m)}$, is the ideal

$$
I^{(m)}=\bigcap_{P \in \operatorname{Ass}(I)}\left(I^{m} R_{P} \cap R\right),
$$

where $R_{P}$ denotes the localization of $R$ at the prime ideal $P$.

Remark 2.4. In the literature, there is some ambiguity concerning the notion of symbolic powers. The intersection in the definition of the symbolic power is sometimes taken over all associated primes and sometimes just over the minimal primes of $I$. In general, these two possible definitions yield different results. However, they agree in the case of radical ideals, thus, in particular, also in the case of squarefree monomial ideals.

We will be concerned with the analysis of generators of minimal degree in the symbolic powers $I^{(m)}$ of $I$. While the general definition of the $m$-th symbolic power of $I$ is based on localization, for squarefree monomial ideals the following result will prove useful.

Theorem 2.5. Suppose that $I \subseteq R$ is a squarefree monomial ideal with minimal primary decomposition $I=P_{1} \cap \cdots \cap P_{s}$. Then for all $m \geq 1$,

$$
I^{(m)}=P_{1}^{m} \cap \cdots \cap P_{s}^{m} .
$$

Proof. This result is a special case of [6, Theorem 3.7].

The next result enables us to determine if a particular monomial belongs to $I^{(m)}$.

Lemma 2.6. Let $I \subseteq R$ be a squarefree monomial ideal with minimal primary decomposition $I=P_{1} \cap P_{2} \cap \cdots \cap P_{s}$ with $P_{j}=\left\langle x_{j_{1}}, \ldots, x_{j_{s_{j}}}\right\rangle$ for $j=1, \ldots$, s. Then $x_{1}^{a_{1}} \cdots x_{n}^{a_{n}} \in I^{(m)}$ if and only if $a_{j_{1}}+\cdots+a_{j_{s_{j}}} \geq m$ for $j=1, \ldots, s$.

Proof. By Theorem 2.5, $I^{(m)}=P_{1}^{m} \cap \cdots \cap P_{s}^{m}$. So $x_{1}^{a_{1}} \cdots x_{n}^{a_{n}} \in I^{(m)}$ if and only if $x_{1}^{a_{1}} \cdots x_{n}^{a_{n}}$ is in $P_{j}^{m}$ for all $j=1, \ldots, s$. This happens if and only if there exists at least one generator $f_{j} \in P_{j}^{m}$ such that $f_{j}$ divides $x_{1}^{a_{1}} \cdots x_{n}^{a_{n}}$ (for $j=1, \ldots, s$ ), which is equivalent to requiring $a_{j_{1}}+\cdots+a_{j_{s_{j}}} \geq m$ for $j=1, \ldots, s$.

2.3. Waldschmidt constants. We complete this section by reviewing some useful results on $\widehat{\alpha}(I)$, the Waldschmidt constant of a homogeneous ideal.

Lemma 2.7 (Subadditivity). Let $I$ be a radical homogeneous ideal in $R=k\left[x_{1}, \ldots, x_{n}\right]$. Then

(i) $\alpha\left(I^{(c+d)}\right) \leq \alpha\left(I^{(c)}\right)+\alpha\left(I^{(d)}\right)$ for all positive $c, d \in \mathbb{N}$.

(ii) $\widehat{\alpha}(I)=\lim _{m \rightarrow \infty} \frac{\alpha\left(I^{(m)}\right)}{m}$ is the infimum of $\alpha\left(I^{(m)}\right) / m$ for $m \in \mathbb{N}$.

Proof. The subadditivity of $\alpha(-)$ is a consequence of the fact that symbolic powers of any radical homogeneous ideal form a graded system, meaning that $I^{(c)} I^{(d)} \subseteq I^{(c+d)}$ for all $c, d \geq 0$ (see e.g [22, Example 2.4.16 (iv)]). The statement in part (ii) then follows from $(i)$ by means of the general principle of subadditivity in [23, Lemma A.4.1]. See [19, Remark III.7] or [3, Lemma 2.3.1] for a version of the result in (ii) and its proof. Alternatively, use Fekete's Lemma [13] as in [1]. 


\section{The Waldschmidt CONSTANT AND A Linear PROGRAM}

When $I$ is a squarefree monomial ideal, we show that $\widehat{\alpha}(I)$ can be expressed as the value to a certain linear program arising from the structure of the associated primes of $I$. For the convenience of the reader, we review the relevant definitions concerning linear programming (we have used [23] for our reference).

A linear program (henceforth LP) is a problem that can be expressed as:

$$
\text { minimize } \mathbf{b}^{T} \mathbf{y}
$$

subject to $A \mathbf{y} \geq \mathbf{c}$ and $\mathbf{y} \geq \mathbf{0}$

where $\mathbf{b}$ is an $s$-vector, $\mathbf{c}$ is an $r$-vector, $\mathbf{0}$ is the zero $r$-vector, and $A$ is an $r \times s$ real coefficient matrix. Here, $\mathbf{d} \geq \mathbf{e}$ denotes the partial order where the $i$-th coordinate entry of $\mathbf{d}$ is larger than the $i$-th coordinate entry of $\mathbf{e}$ for all $i$. Note that we wish to solve for the $s$-vector $\mathbf{y}$. The equation $\mathbf{b}^{T} \mathbf{y}$ is the constraint equation. Any $\mathbf{y}$ that satisfies $A \mathbf{y} \geq \mathbf{c}$ and $\mathbf{y} \geq \mathbf{0}$ is called a feasible solution. If $\mathbf{y}^{*}$ is a feasible solution that optimizes the constraint equation, then $\mathbf{b}^{T} \mathbf{y}^{*}$ is the value of LP. Associated to the LP ( $\star$ ) is its dual linear program:

$$
\begin{aligned}
\operatorname{maximize} & \mathbf{c}^{T} \mathbf{x} \\
\text { subject to } & A^{T} \mathbf{x} \leq \mathbf{b} \text { and } \mathbf{x} \geq \mathbf{0}
\end{aligned}
$$

A fundamental result in linear programming is that both a linear program and its dual have the exact same value, i.e., $\mathbf{c}^{T} \mathbf{x}^{*}=\mathbf{b}^{T} \mathbf{y}^{*}$ (see [23, Theorem A.3.1]). In particular, we shall find the following fact useful.

Lemma 3.1. Consider the $L P$

$$
\text { minimize } \mathbf{b}^{T} \mathbf{y}
$$

subject to $A \mathbf{y} \geq \mathbf{c}$ and $\mathbf{y} \geq \mathbf{0}$

and suppose that $\mathbf{y}^{*}$ is the feasible solution that gives the value of this LP. If $\mathbf{x}$ is any feasible solution of the associated dual LP, then $\mathbf{c}^{T} \mathbf{x} \leq \mathbf{b}^{T} \mathbf{y}^{*}$.

Proof. For any feasible solution $\mathbf{x}$, we have

$$
\mathbf{c}^{T} \mathbf{x}=\mathbf{x}^{T} \mathbf{c} \leq \mathbf{x}^{T} A \mathbf{y}^{*}=\left(A^{T} \mathbf{x}\right)^{T} \mathbf{y}^{*} \leq \mathbf{b}^{T} \mathbf{y}^{*} .
$$

We now have the machinery to state and prove the first main result of this paper.

Theorem 3.2. Let $I \subseteq R$ be a squarefree monomial ideal with minimal primary decomposition $I=P_{1} \cap P_{2} \cap \cdots \cap P_{s}$ with $P_{j}=\left\langle x_{j_{1}}, \ldots, x_{j_{j}}\right\rangle$ for $j=1, \ldots$, s. Let $A$ be the $s \times n$ matrix where

Consider the following LP:

$$
A_{i, j}= \begin{cases}1 & \text { if } x_{j} \in P_{i} \\ 0 & \text { if } x_{j} \notin P_{i}\end{cases}
$$

$$
\text { minimize } \mathbf{1}^{T} \mathbf{y}
$$

subject to $A \mathbf{y} \geq \mathbf{1}$ and $\mathbf{y} \geq \mathbf{0}$

and suppose that $\mathbf{y}^{*}$ is a feasible solution that realizes the optimal value. Then

$$
\widehat{\alpha}(I)=\mathbf{1}^{T} \mathbf{y}^{*} \text {. }
$$


That is, $\widehat{\alpha}(I)$ is the value of the $L P$.

Proof. Suppose that $\left(\mathbf{y}^{*}\right)^{T}=\left[\begin{array}{llll}y_{1}^{*} & y_{2}^{*} & \cdots & y_{n}^{*}\end{array}\right]$ is the feasible solution that realizes the optimal solution to the LP. Because the entries of $\mathbf{y}^{*}$ are rational numbers, we can write $\left(\mathbf{y}^{*}\right)^{T}=\left[\begin{array}{llll}\frac{a_{1}}{b_{1}} & \frac{a_{2}}{b_{2}} & \cdots & \frac{a_{n}}{b_{n}}\end{array}\right]$ with integers $a_{i}, b_{i}$ for $i=1, \ldots, n$.

Set $b=\operatorname{lcm}\left(b_{1}, \ldots, b_{n}\right)$. Then $A(b \mathbf{y}) \geq \mathbf{b}$ where $\mathbf{b}$ is an $s$-vector of $b$ 's. So, $(b \mathbf{y})$ is a feasible integer solution to the system $A \mathbf{z} \geq \mathbf{b}$. In other words, for each $j=1, \ldots, s$,

$$
b\left(\frac{a_{j_{1}}}{b_{j_{1}}}+\cdots+\frac{a_{j_{s_{j}}}}{b_{j_{s_{j}}}}\right)=\frac{b a_{j_{1}}}{b_{j_{1}}}+\cdots+\frac{b a_{j_{s_{j}}}}{b_{j_{s_{j}}}} \geq b .
$$

It then follows by Lemma 2.6 that

$$
x_{1}^{\frac{b a_{1}}{b_{1}}} x_{2}^{\frac{b a_{2}}{b_{2}}} \cdots x_{n}^{\frac{b a_{n}}{b_{n}}} \in I^{(b)} .
$$

Thus,

or equivalently (by Lemma 2.7),

$$
\alpha\left(I^{(b)}\right) \leq \frac{b a_{1}}{b_{1}}+\frac{b a_{2}}{b_{2}}+\cdots+\frac{b a_{n}}{b_{n}},
$$

$$
\widehat{\alpha}(I) \leq \frac{\alpha\left(I^{(b)}\right)}{b} \leq \frac{a_{1}}{b_{1}}+\frac{a_{2}}{b_{2}}+\cdots+\frac{a_{n}}{b_{n}}=\mathbf{1}^{T} \mathbf{y}^{*} .
$$

To show the reverse inequality, suppose for a contradiction that $\widehat{\alpha}(I)<\mathbf{1}^{T} \mathbf{y}^{*}$. By Lemma 2.7 we have $\widehat{\alpha}(I)=\inf \left\{\alpha\left(I^{(m)}\right) / m\right\}_{m \in \mathbb{N}}$. In particular, there must exist some $m$ such that

$$
\frac{\alpha\left(I^{(m)}\right)}{m}<\frac{a_{1}}{b_{1}}+\frac{a_{2}}{b_{2}}+\cdots+\frac{a_{n}}{b_{n}}=\mathbf{1}^{T} \mathbf{y}^{*} .
$$

Let $x_{1}^{e_{1}} x_{2}^{e_{2}} \cdots x_{n}^{e_{n}} \in I^{(m)}$ be a monomial with $e_{1}+\cdots+e_{n}=\alpha\left(I^{(m)}\right)$. Then, by Lemma 2.6, we have

$$
e_{j_{1}}+\cdots+e_{j_{s_{j}}} \geq m \text { for all } j=1, \ldots, s .
$$

In particular, if we divide all the $s$ equations by $m$, we have

$$
\frac{e_{j_{1}}}{m}+\cdots+\frac{e_{j_{s_{j}}}}{m} \geq 1 \text { for all } j=1, \ldots, s .
$$

But then

$$
\mathbf{w}^{T}=\left[\begin{array}{lll}
\frac{e_{1}}{m} & \cdots & \frac{e_{s}}{m}
\end{array}\right]^{T}
$$

satisfies $A \mathbf{w} \geq \mathbf{1}$ and $\mathbf{w} \geq \mathbf{0}$. In other words, $\mathbf{w}$ is a feasible solution to the LP, and furthermore, $\frac{\alpha\left(I^{(m)}\right)}{m}=\mathbf{1}^{T} \mathbf{w}<\frac{a_{1}}{b_{1}}+\frac{a_{2}}{b_{2}}+\cdots+\frac{a_{n}}{b_{n}}=\mathbf{1}^{T} \mathbf{y}^{*}$, contradicting the fact that $\mathbf{1}^{T} \mathbf{y}^{*}$ is the value of the LP.

Remark 3.3. The set of feasible solutions of the LP in Theorem 3.2 is the symbolic polyhedron for the monomial ideal $I$ as defined in [6, Definition 5.3]:

$$
\mathcal{Q}=\bigcap_{P \in \max \operatorname{Ass}(I)} \operatorname{conv} \mathcal{L}\left(Q_{\subseteq P}\right)
$$

Here, $Q_{\subseteq P}$ is the intersection of all primary ideals $Q_{i}$ in the primary decomposition of $I$ with $\sqrt{Q_{i}} \subseteq P, \mathcal{L}\left(Q_{\subseteq P}\right)$ is the set of lattice points $a \in \mathbb{N}^{n}$ with $x^{a}=x_{1}^{a_{1}} \cdots x_{n}^{a_{n}} \in Q_{\subseteq P}$, 
THE WALDSCHMIDT CONSTANT FOR SQUAREFREE MONOMIAL IDEALS

and $\operatorname{conv}(-)$ denotes the convex hull. When $I$ is a squarefree monomial ideal $I$, then $\operatorname{Ass}(I)=\max \operatorname{Ass}(I)$ and $Q_{\subseteq P}=P$ for any $P \in \operatorname{Ass}(I)$. So we have $\mathcal{L}\left(Q_{\subseteq P}\right)=\mathcal{L}(P)=$ $\left\{\mathbf{x} \mid \mathbf{x} \geq \mathbf{0}, a_{i} \cdot \mathbf{x} \geq 1\right\}$, where $a_{i}$ is the $i$-th row of the matrix $A$ in Theorem 3.2, Clearly then $\bigcap_{i} \mathcal{L}\left(Q_{\subseteq P}\right)=\{\mathbf{x} \mid \mathbf{x} \geq \mathbf{0}, A \mathbf{x} \geq \mathbf{1}\}$.

Furthermore, the optimal value of our LP is the same as $\alpha(\mathcal{Q})$ as defined in [6] before Corollary 6.2, thus Theorem 3.2 is a (useful!) restatement (with easier proof) of [6, Corollary 6.3].

Remark 3.4. Because the set of optimal solutions to an integer LP consists of points with rational coordinates, Theorem 3.2 allows us to conclude that the Waldschmidt constant of any squarefree monomial ideal is rational. The same is true for arbitrary monomial ideals by making use of the symbolic polyhedron described above.

\section{The Waldschmidt CONSTANT In TERms of a FRACTIONAL CHROMATiC Number}

As shown in the last section, the Waldschmidt constant $\widehat{\alpha}(I)$ of a squarefree monomial ideal $I \subseteq R=k\left[x_{1}, \ldots, x_{n}\right]$ is the optimal value of a linear program. On the other hand, a squarefree monomial ideal can also be viewed as the edge ideal of a hypergraph $H=(V, E)$ where $V=\left\{x_{1}, \ldots, x_{n}\right\}$ and $\left\{x_{i_{1}}, \ldots, x_{i_{t}}\right\} \in E$ is an edge if and only if $x_{i_{1}} \cdots x_{i_{t}}$ is a minimal generator of $I$. We now show that $\widehat{\alpha}(I)$ can be expressed in terms of a combinatorial invariant of $H$, specifically, the fractional chromatic number of $H$. Recall that we are assuming that all our hypergraphs $H=(V, E)$ have a non-trivial edge.

We begin by defining the fractional chromatic number of a hypergraph $H=(V, E)$. Set

$$
\mathcal{W}=\{W \subseteq V \mid W \text { is an independent set of } H\}
$$

Definition 4.1. Let $H=(V, E)$ be a hypergraph. Suppose that $\mathcal{W}=\left\{W_{1}, \ldots, W_{t}\right\}$ is the set of all independent sets of $H$. Let $B$ be the $n \times t$ matrix given by

$$
B_{i, j}= \begin{cases}1 & \text { if } x_{i} \in W_{j} \\ 0 & \text { if } x_{i} \notin W_{j} .\end{cases}
$$

The optimal value of the following LP, denoted $\chi^{*}(H)$,

minimize $y_{W_{1}}+y_{W_{2}}+\cdots+y_{W_{t}}=\mathbf{1}^{T} \mathbf{y}$

subject to $B \mathbf{y} \geq \mathbf{1}$ and $\mathbf{y} \geq \mathbf{0}$

is the fractional chromatic number of the hypergraph $H$.

Remark 4.2. If $H=(V, E)$ is a hypergraph with a non-trivial edge, then $\chi^{*}(H) \neq 1$.

Remark 4.3. A colouring of a hypergraph $H=(V, E)$ is an assignment of a colour to every $x \in V$ so that no edge is mono-coloured. The minimum number of colours needed to give $H$ a valid colouring is the chromatic number of $H$, and is denoted $\chi(H)$. The value of $\chi(H)$ can also be interpreted as the value of the optimal integer solution to the LP in the previous definition. In other words, the fractional chromatic number is the relaxation of the requirement that the previous LP have integer solutions.

We next give a lemma which may be of independent interest due to its implications on computing fractional chromatic numbers. Our lemma shows that the dual of the LP 
that defines the fractional chromatic number can be reformulated in terms of a smaller number of constraints.

Lemma 4.4. Let $H=(V, E)$ be a hypergraph. Suppose that $\mathcal{W}^{\prime}=\left\{W_{1}, \ldots, W_{s}\right\}$ is the set of all maximal independent sets of $H$. Let $B^{\prime}$ be the $n \times s$ matrix given by

$$
B_{i, j}^{\prime}= \begin{cases}1 & \text { if } x_{i} \in W_{j} \\ 0 & \text { if } x_{i} \notin W_{j} .\end{cases}
$$

and let $B$ be the matrix defined in 4.1. Then the following two linear programs have the same feasible solution sets and the same optimal values:

$$
\begin{array}{llll}
\text { maximize } & w_{1}+\cdots+w_{n}=\mathbf{1}^{T} \mathbf{w} & \text { maximize } & w_{1}+\cdots+w_{n}=\mathbf{1}^{T} \mathbf{w} \\
\text { subject to } & B^{T} \mathbf{w} \leq \mathbf{1} \text { and } \mathbf{w} \geq \mathbf{0} & \text { subject to } & B^{\prime T} \mathbf{w} \leq \mathbf{1} \text { and } \mathbf{w} \geq \mathbf{0}
\end{array}
$$

In particular, the fractional chromatic number $\chi^{*}(H)$ can also be computed as the optimal value of the second linear program.

Proof. It is clear from the definitions that there is a block decomposition

$$
B=\left[\begin{array}{ll}
B^{\prime} & C
\end{array}\right]
$$

where $C$ is a $n \times(t-s)$ matrix corresponding to non-maximal independent sets. The feasible set for the first LP is thus given by the constraints $B^{\prime T} \mathbf{w} \leq \mathbf{1}, C^{T} \mathbf{w} \leq \mathbf{1}$ and $\mathbf{w} \geq \mathbf{0}$. It is clear that any feasible solution of the first LP is also feasible for the second. For the converse we need to observe that the constraint equations $C^{T} \mathbf{w} \leq \mathbf{1}$ are all redundant. To see why, note that any row in $C^{T}$ corresponds to a non-maximal independent set $W^{\prime}$. So, there is a maximal independent set $W$ such that $W^{\prime} \subseteq W$, and if $\mathbf{w}$ satisfies the constraint corresponding to the row $W$, it will also have to satisfy the constraint coming from the row corresponding to $W^{\prime}$. In particular, this tells us that $B^{\prime T} \mathbf{w} \leq \mathbf{1}$ implies $C^{T} \mathbf{w} \leq \mathbf{1}$, and consequently the two LPs have the same feasible sets. Since the LPs also have the same objective function, their optimal values will be the same. Since the first LP is the dual of the LP in Definition 4.1, the common value of these LPs is equal to $\chi^{*}(H)$.

Our goal is now to show that if $I$ is any squarefree monomial ideal of $R$, and if $H$ is the hypergraph such that $I=I(H)$, then $\widehat{\alpha}(I)$ can be expressed in terms of $\chi^{*}(H)$. To do this, we relate the matrix $A$ with the matrix $B^{\prime}$ of Lemma 4.4.

Lemma 4.5. Let $H=(V, E)$ be a hypergraph with edge ideal $I=I(H)$. Suppose that $W=\left\{W_{1}, \ldots, W_{s}\right\}$ are the maximal independent sets of $H$. Let $A$ be the $s \times n$ matrix of the LP of Theorem 3.2 constructed from $I(H)$, and let $B^{\prime}$ be the $n \times s$ matrix of Lemma 4.4. Then

$$
B^{\prime}=(\mathbb{I}-A)^{T} \text { and } A=\left(\mathbb{I}-B^{\prime}\right)^{T}
$$

where $\mathbb{I}$ denotes an appropriate sized matrix with every entry equal to one.

Proof. By Lemma 2.2, a set of variables generates a minimal prime ideal containing $I$ if and only if its complement is a maximal independent set of $H$, i.e., there is a one-toone correspondence between the associated primes $P_{1}, \ldots, P_{s}$ of $I(H)$ and the maximal 
independent sets of $H$. This complementing is represented by the formula $\mathbb{I}-A$ or $\mathbb{I}-B^{\prime}$, while transposition occurs since the variables index rows for $B^{\prime}$ and columns for $A$.

Theorem 4.6. Suppose that $H=(V, E)$ is a hypergraph with a non-trivial edge, and let $I=I(H)$. Then

$$
\widehat{\alpha}(I)=\frac{\chi^{*}(H)}{\chi^{*}(H)-1} .
$$

Proof. Consider LP introduced in Lemma 4.4, namely

maximize $w_{1}+\cdots+w_{n}=\mathbf{1}^{T} \mathbf{w}$

subject to $B^{\prime T} \mathbf{w} \leq \mathbf{1}$ and $\mathbf{w} \geq \mathbf{0}$.

By Lemma 4.4, the optimal value of this LP is $\chi^{*}(H)$. Let $\mathbf{w}^{*}$ denote an optimal solution for this LP. We claim that $\frac{1}{\chi^{*}(H)-1} \mathbf{w}^{*}$ is a feasible solution for the LP defining $\widehat{\alpha}(I)$. Indeed, using Lemma 4.5, we have

$$
\begin{aligned}
\mathbf{1} & =\frac{1}{\chi^{*}(H)-1}\left(\chi^{*}(H) \mathbf{1}-\mathbf{1}\right) \leq \frac{1}{\chi^{*}(H)-1}\left(\mathbb{I} \mathbf{w}^{*}-B^{\prime T} \mathbf{w}^{*}\right) \\
& =\frac{1}{\chi^{*}(H)-1}\left(\mathbb{I}-B^{\prime}\right)^{T} \mathbf{w}^{*}=\frac{1}{\chi^{*}(H)-1} A \mathbf{w}^{*} .
\end{aligned}
$$

In particular, $\mathbf{1} \leq A\left(\frac{1}{\chi^{*}(H)-1} \mathbf{w}^{*}\right)$, where $\mathbf{1}$ is an appropriate sized vector of 1 's. Thus

$$
\widehat{\alpha}(I) \leq \frac{1}{\chi^{*}(H)-1}\left(w_{1}+\cdots+w_{n}\right)=\frac{\chi^{*}(H)}{\chi^{*}(H)-1} .
$$

A similar computation shows that, if $\mathbf{y}^{*}$ is the optimal solution for the LP

minimize $y_{1}+\cdots+y_{n}=\mathbf{1}^{T} \mathbf{y}$

subject to $A \mathbf{y} \geq \mathbf{1}$ and $\mathbf{y} \geq \mathbf{0}$

that is, $\mathbf{1}^{T} \mathbf{y}^{*}=\widehat{\alpha}(I)$, then $\frac{1}{\widehat{\alpha}(I)-1} \mathbf{y}^{*}$ is a feasible solution for the dual LP described in the beginning of this proof. Indeed, using Lemma 4.5 we have

$$
\begin{aligned}
B^{\prime T}\left(\frac{1}{\widehat{\alpha}(I)-1} \mathbf{y}^{*}\right) & =(\mathbb{I}-A)\left(\frac{1}{\widehat{\alpha}(I)-1} \mathbf{y}^{*}\right) \\
& =\frac{1}{\widehat{\alpha}(I)-1}(\mathbb{I}-A) \mathbf{y}^{*}=\frac{1}{\widehat{\alpha}(I)-1}\left(\widehat{\alpha}(I) \mathbf{1}-A \mathbf{y}^{*}\right) .
\end{aligned}
$$

Because $A \mathbf{y}^{*} \geq \mathbf{1}$, we now have $B^{\prime T}\left(\frac{1}{\widehat{\alpha}(I)-1} \mathbf{y}^{*}\right) \leq \mathbf{1}$. Thus Lemma 3.1 yields the inequality

$$
\chi^{*}(H) \geq \frac{1}{\widehat{\alpha}(I)-1}\left(y_{1}+\cdots+y_{n}\right)=\frac{\widehat{\alpha}(I)}{\widehat{\alpha}(I)-1}
$$

and by elementary manipulations this inequality is equivalent to $\widehat{\alpha}(I) \geq \frac{\chi^{*}(H)}{\chi^{*}(H)-1}$.

We end this section with an application that illustrates the power of Theorem 4.6. 
THE WALDSCHMIDT CONSTANT FOR SQUAREFREE MONOMIAL IDEALS

Corollary 4.7. Suppose that $I$ and $J$ are two squarefree monomial ideals of the ring $R=k\left[x_{1}, \ldots, x_{n}, y_{1}, \ldots, y_{n}\right]$. Furthermore, suppose that $I$ is generated by monomials only in the $x_{i}$ 's and $J$ is generated by monomials only in the $y_{j}$ 's. Then

$$
\widehat{\alpha}(I+J)=\min \{\widehat{\alpha}(I), \widehat{\alpha}(J)\} .
$$

Proof. We can view $I$ as the edge ideal of a hypergraph $H$ on the vertices $\left\{x_{1}, \ldots, x_{n}\right\}$ and $J$ as the edge ideal of a hypergraph $K$ on the vertices $\left\{y_{1}, \ldots, y_{n}\right\}$. Thus $I+J$ is the edge ideal of the hypergraph $H \cup K$ where $H$ and $K$ are disjoint. But then

$$
\chi^{*}(H \cup K)=\max \left\{\chi^{*}(H), \chi^{*}(K)\right\},
$$

which is equivalent to the statement

$$
\frac{\chi^{*}(H \cup K)}{\chi^{*}(H \cup K)-1}=\min \left\{\frac{\chi^{*}(H)}{\chi^{*}(H)-1}, \frac{\chi^{*}(K)}{\chi^{*}(K)-1}\right\} .
$$

Now apply Theorem 4.6.

\section{A Chudnovsky-Like LOWER Bound On $\widehat{\alpha}(I)$}

Chudnovsky [5] first proposed a conjectured lower bound on $\widehat{\alpha}(I)$ when $I$ is the ideal of a set of points in a projective space. Motivated by this conjecture, Cooper, et al. [6] formulated an analogous conjecture for all monomial ideals. Recall that the big height of $I$, denoted big-height $(I)$, is the maximum of the heights of $P \in \operatorname{Ass}(I)$.

Conjecture 5.1 ([6, Conjecture 6.6]). Let I be a monomial ideal with big-height $(I)=e$. Then

$$
\widehat{\alpha}(I) \geq \frac{\alpha(I)+e-1}{e} .
$$

Remark 5.2. In the original formulation, the authors make a conjecture about $\alpha(\mathcal{Q})$ of the symbolic polyhedron $\mathcal{Q}$ of $I$ as introduced in Remark 3.3. It is enough to know that in our context, $\alpha(\mathcal{Q})=\widehat{\alpha}(I)$.

By taking the viewpoint that $\widehat{\alpha}(I)$ is the solution to a LP, we are able to verify the above conjecture for all squarefree monomial ideals.

Theorem 5.3. Let $I$ be a squarefree monomial ideal with big-height $(I)=e$. Then

$$
\widehat{\alpha}(I) \geq \frac{\alpha(I)+e-1}{e} .
$$

Proof. By Theorem 3.2, $\widehat{\alpha}(I)$ is the optimum value of the LP that asks to minimize $y_{1}+\cdots+y_{n}$ subject to the constraints $A \mathbf{y} \geq \mathbf{1}$ and $\mathbf{y} \geq \mathbf{0}$, with $A$ obtained from the primary decomposition of $I$. It is enough to show that any feasible solution $\mathbf{y}$ for this LP satisfies

$$
\sum_{i=1}^{n} y_{i} \geq \frac{\alpha(I)+e-1}{e}
$$

in order to conclude that the optimal solution satisfies the same inequality, hence the optimal value of the program satisfies the desired inequality $\widehat{\alpha}(I) \geq \frac{\alpha(I)+e-1}{e}$. 
Let $I=P_{1} \cap P_{2} \cap \cdots \cap P_{s}$ be the primary decomposition for $I$, where the $P_{i}$ are prime ideals generated by a subset of the variables. Since $P_{1} P_{2} \cdots P_{s} \subseteq I$, we must have $\alpha\left(P_{1} P_{2} \cdots P_{s}\right) \geq \alpha(I)$, hence $s \geq \alpha(I)$. The feasible set of the above LP is thus defined by at least $\alpha(I)$ inequalities. Since big-height $(I)=e$, each of these inequalities involves at most $e$ of the variables. Both of these observations will be used in the proof.

Let $\mathbf{y}$ be a feasible solution for the above LP. If $\alpha(I)=1$, then because any constraint equation implies $y_{1}+\cdots+y_{n} \geq 1$, the inequality $\sum_{i=1}^{n} y_{i} \geq \frac{\alpha(I)+e-1}{e}=1$ is satisfied. So, we can assume that $\alpha(I) \geq 2$.

We will show that there exist distinct indices $k_{1}, \ldots, k_{\alpha(I)-1}$ so that $y_{k_{i}} \geq \frac{1}{e}$ for $1 \leq$ $i \leq \alpha(I)-1$. The proof of this claim is by induction. For the base case, we need to find one index $k_{1}$ such that $y_{k_{1}} \geq \frac{1}{e}$. Let $y_{i_{1}}+\cdots+y_{i_{e}} \geq 1$ be the constraint equation constructed from the height $e$ associated prime. Since $\mathbf{y}$ is a feasible solution, at least one of $y_{i_{1}}, \ldots, y_{i_{e}}$ must be $\geq \frac{1}{e}$. Let $k_{1}$ be the corresponding index. This proves our base case.

Now let $1<j \leq \alpha(I)-1$ and suppose that there exist pairwise distinct indices $k_{1}, \ldots, k_{j-1}$ so that $y_{k_{i}} \geq \frac{1}{e}$ for $1 \leq i \leq j-1$. Note that the monomial $x_{k_{1}} x_{k_{2}} \cdots x_{k_{j-1}}$ of degree $j-1 \leq \alpha(I)-2$ is not an element of $I$. Consequently there exists a prime $P_{\ell}$ among the associated primes of $I$ that does not contain the monomial $x_{k_{1}} x_{k_{2}} \cdots x_{k_{j-1}}$, thus $P_{\ell}$ contains none of the variables $x_{k_{1}}, x_{k_{2}}, \ldots, x_{k_{j-1}}$. Consider the inequality of the LP corresponding to the prime $P_{\ell}$

$$
y_{\ell_{1}}+y_{\ell_{2}}+\cdots+y_{\ell_{s_{\ell}}} \geq 1
$$

This inequality involves at most $e$ of the entries of $\mathbf{y}$, thus $y_{\ell_{t}} \geq \frac{1}{e}$ for some $t$. Since $x_{\ell_{t}} \in P_{\ell}$ and none of the variables $x_{k_{1}}, x_{k_{2}}, \ldots x_{k_{j-1}}$ are in $P_{\ell}$, we conclude that $\ell_{t}$ must be distinct from any of the indices $k_{1}, \ldots, k_{j-1}$. Setting $k_{j}=\ell_{t}$ gives a pairwise distinct set of indices $k_{1}, \ldots, k_{j}$ so that $y_{k_{i}} \geq \frac{1}{e}$ for $1 \leq i \leq j$. This finishes the proof of our claim.

Now consider the monomial $x_{k_{1}} x_{k_{2}} \cdots x_{k_{\alpha(I)-1}}$, which has degree $\alpha(I)-1$ and consequently is not an element of $I$. Then there exists an associated prime $P_{u}$ of $I$ so that none of the variables $x_{k_{1}}, x_{k_{2}}, \ldots, x_{k_{\alpha(I)-1}}$ are in $P_{u}$. The inequality in the LP corresponding to the prime $P_{u}$

$$
y_{u_{1}}+y_{u_{2}}+\cdots+y_{u_{s_{u}}} \geq 1
$$

together with the previously established inequalities

$$
y_{k_{1}} \geq \frac{1}{e}, y_{k_{2}} \geq \frac{1}{e}, \ldots, y_{k_{\alpha(I)-1}} \geq \frac{1}{e}
$$

and the non-negativity conditions $y_{i} \geq 0$ for $1 \leq i \leq n$ yield

$$
\begin{aligned}
\sum_{i=1}^{n} y_{i} & \geq y_{k_{1}}+y_{k_{2}}+\ldots+y_{k_{\alpha(I)-1}}+y_{u_{1}}+y_{u_{2}}+\ldots+y_{u_{s_{u}}} \\
& \geq \frac{\alpha(I)-1}{e}+1=\frac{\alpha(I)+e-1}{e} .
\end{aligned}
$$

The first inequality also uses the fact that $\left\{k_{1}, k_{2}, \ldots, k_{\alpha(I)-1}\right\} \cap\left\{u_{1}, u_{2}, \ldots, u_{s_{u}}\right\}=\varnothing$. 
THE WALDSCHMIDT CONSTANT FOR SQUAREFREE MONOMIAL IDEALS

Since $\widehat{\alpha}(I)=\sum_{i=1}^{n} y_{i}^{*}$ for some feasible solution $\mathbf{y}^{*}$ of the LP, we now have

$$
\widehat{\alpha}(I)=\sum_{i=1}^{n} y_{i}^{*} \geq \frac{\alpha(I)+e-1}{e} .
$$

Remark 5.4. The lower bound in the above theorem is optimal; see Theorem 7.5 and Remark 7.6.

\section{The Waldschmidt COnstant FOR EDGE IDEALS}

In this section, we apply our methods to examine the Waldschmidt constant for edge ideals for several families of finite simple graphs, and relate this algebraic invariant to invariants of the graph.

In the following, let $G=(V, E)$ be a finite simple graph with vertex set $V=\left\{x_{1}, \ldots, x_{n}\right\}$ and edge set $E$. Let $k$ be a field and set $R=k\left[x_{1}, \ldots x_{n}\right]$. The edge ideal of $G$ is then the squarefree quadratic monomial ideal

$$
I(G)=\left\langle x_{i} x_{j} \mid\left\{x_{i}, x_{j}\right\} \in E\right\rangle \subseteq R,
$$

i.e., this is the special case of an edge ideal first introduced in Section 2. All terminology in that section can therefore be applied to graphs. In particular, the notion of vertex cover specializes to graphs as well as the correspondence outlined in Lemma 2.2 which gives a bijection between minimal associated primes of $I(G)$ and minimal vertex covers of $G$.

Definition 6.1. A $k$-colouring for $G$ is an assignment of $k$ labels (or colours) to the elements of $V$ so that no two adjacent vertices are given the same label. The chromatic number of $G, \chi(G)$, is the smallest integer $k$ so that $G$ admits a $k$-colouring.

Definition 6.2. A clique of $G$ is a set of pairwise adjacent vertices of $G$. A maximum clique of $G$ is a clique such that $G$ admits no clique with more vertices. The clique number $\omega(G)$ is the number of vertices in a maximum clique in $G$.

We obtain the following bound on $\widehat{\alpha}(I(G))$ in terms of these invariants.

Theorem 6.3. Let $G$ be a non-empty graph with chromatic number $\chi(G)$ and clique number $\omega(G)$. Then

$$
\frac{\chi(G)}{\chi(G)-1} \leq \widehat{\alpha}(I(G)) \leq \frac{\omega(G)}{\omega(G)-1} .
$$

Proof. The fractional chromatic number $\chi^{*}(G)$ of the graph $G$ is the solution to the LP of Defintion 4.1. Now $\chi(G)$ is the integer solution to this LP, while $\omega(G)$ is the integer solution to the dual of this LP. This implies that $\omega(G) \leq \chi^{*}(G) \leq \chi(G)$, and so the result follows from Theorem 4.6 which gives $\widehat{\alpha}(I(G))=\frac{\chi^{*}(G)}{\chi^{*}(G)-1}$.

The above lower bound improves the lower bound from Theorem 5.3 , 
Theorem 6.4. Let $I(G)$ be the edge ideal of a graph $G$ and let big-height $(I(G))=e$. Then

$$
\widehat{\alpha}(I(G)) \geq \frac{\chi(G)}{\chi(G)-1} \geq \frac{e+1}{e}=\frac{\alpha(I(G))+e-1}{e} .
$$

Proof. Theorem 6.3 already shows the first inequality, so it suffices to verify the second inequality $\chi(G) /(\chi(G)-1) \geq(e+1) / e$.

Let $P$ be the associated prime of $I(G)$ with height $e$. If $P=\left\langle x_{i_{1}}, \ldots, x_{i_{e}}\right\rangle$, then $W=\left\{x_{1}, \ldots, x_{n}\right\} \backslash\left\{x_{i_{1}}, \ldots, x_{i_{e}}\right\}$ is an independent set of $G$. We can now colour $G$ with $e+1$ colours by colouring the vertices of $W$ one colour, and then colour each vertex of $\left\{x_{i_{1}}, \ldots, x_{i_{e}}\right\}$ with a distinct colour. So $\chi(G) \leq e+1$, which gives the result.

We now turn to the computation of the Waldschmidt constant for various families of simple graphs. In particular, we examine perfect graphs, $k$-partite graphs, cycles, and complements of cycles. We will use these results to give a simplified proof to a result of Bocci and Franci [2, 14].

We now recall the definitions of the family of graphs we wish to study. If $G=(V, E)$ is a graph and $A \subseteq V$, then the induced subgraph of $G$ on $A$, denoted $G_{A}$, is the graph $G_{A}=\left(A, E_{A}\right)$ where $E_{A}=\{e \in E \mid e \subseteq A\}$. We say a graph $G$ is perfect if $\omega\left(G_{A}\right)=\chi\left(G_{A}\right)$ for all $A \subseteq V$. A graph $G=(V, E)$ is a $k$-partite graph if there exists a $k$-paritition $V=V_{1} \cup \cdots \cup V_{k}$ such that no $e \subseteq V_{i}$ for any $i$. When $k=2$, we call $G$ bipartite. The complete $k$-partite graph is a graph with $k$-paritition $V=V_{1} \cup \cdots \cup V_{k}$ and all edges of the form $\left\{v_{i}, v_{j}\right\}$ with $v_{i} \in V_{i}$ and $v_{j} \in V_{j}$ and $i \neq j$. The complete graph on $n$ vertices, denoted $K_{n}$, is the graph on the vertex set $V=\left\{x_{1}, \ldots, x_{n}\right\}$ and edge set $\left\{\left\{x_{i}, x_{j}\right\} \mid 1 \leq i<j \leq n\right\}$. The cycle on $n$ vertices, denoted $C_{n}$, is a graph on $V=$ $\left\{x_{1}, \ldots, x_{n}\right\}$ and edge set $\left\{\left\{x_{1}, x_{2}\right\},\left\{x_{2}, x_{3}\right\}, \ldots,\left\{x_{n-1}, x_{n}\right\},\left\{x_{n}, x_{1}\right\}\right\}$. The complement of a graph $G=(V, E)$, denoted $G^{c}$, is the graph with the same vertex set as $G$, but edge set $\left\{\left\{x_{i}, x_{j}\right\} \mid\left\{x_{i}, x_{j}\right\} \notin E\right\}$.

We will use the following result to compute (or bound) $\chi^{*}(G)$.

Definition 6.5. A graph $G$ is vertex-transitive if for all $u, v \in V(G)$ there is an automorphism $\pi$ of $G$ with $\pi(u)=v$.

Theorem 6.6 ([23, Proposition 3.1.1]). If $G$ is any graph, then $\chi^{*}(G) \geq \frac{|V(G)|}{\alpha(G)}$, where $\alpha(G)$ is the independence number of $G$ (i.e. the size of the largest independent set in $G$ ). Equality holds if $G$ is vertex-transitive.

Examples of vertex-transitive graphs are complete graphs, cycles, and their complements. We are now able to compute $\widehat{\alpha}(I(G))$ for a large number of families of graphs.

Theorem 6.7. Let $G$ be a non-empty graph.

(i) If $\chi(G)=\omega(G)$, then $\widehat{\alpha}(I(G))=\frac{\chi(G)}{\chi(G)-1}$. In particular, this equality holds for all perfect graphs.

(ii) If $G$ is $k$-partite, then $\widehat{\alpha}(I(G)) \geq \frac{k}{k-1}$. In particular, if $G$ is a complete $k$-partite graph, then $\widehat{\alpha}(I(G))=\frac{k}{k-1}$.

(iii) If $G$ is bipartite, then $\widehat{\alpha}(I(G))=2$. 
(iv) If $G=C_{2 n+1}$ is an odd cycle, then $\widehat{\alpha}\left(I\left(C_{2 n+1}\right)\right)=\frac{2 n+1}{n+1}$.

(v) If $G=C_{2 n+1}^{c}$, then $\widehat{\alpha}(I(G))=\frac{2 n+1}{2 n-1}$.

Proof. (i) This result follows immediately from Theorem 6.3. Note that perfect graphs have the property that $\omega(G)=\chi(G)$.

(ii). If $G$ is a $k$-partite graph, then $\chi(G) \leq k$; indeed, if $V=V_{1} \cup \cdots \cup V_{k}$ is the $k$-parititon, colouring all the vertices of $V_{i}$ the same colour gives a valid colouring. By Theorem [6.3, $\widehat{\alpha}(I(G)) \geq \frac{\chi(G)}{\chi(G)-1} \geq \frac{k}{k-1}$. If $G$ is a complete $k$-partite graph, then $\chi(G) \leq$ $k=\omega(G)$ and the desired equality follows by a direct application of Theorem 6.3.

(iii) For any bipartite graph $G, \chi(G)=\omega(G)=2$, so apply $(i)$.

(iv) For an odd cycle $C_{2 n+1}, \chi^{*}\left(C_{2 n+1}\right)=2+1 / n$ by Theorem 6.6. Now apply Theorem 4.6 .

$(v)$ For the complement $G$ of $C_{2 n+1}, \chi^{*}(G)=\frac{2 n+1}{2}$ by Theorem 6.6. Again, apply Theorem 4.6.

Remark 6.8. The fact that $\widehat{\alpha}(I(G))=2$ when $G$ is bipartite is well-known. In fact, the much stronger result that $I(G)^{(m)}=I(G)^{m}$ for all $m$ holds when $G$ is bipartite (see [25]).

Bocci and Franci [2] recently computed the Waldschmidt constant of the StanleyReisner ideal of the so-called $n$-bipyramid. We illustrate the strength of our new techniques by giving a simplified proof of their main result using the above results.

Definition 6.9. The bipyramid over a polytope $P$, denoted $\operatorname{bipyr}(P)$, is the convex hull of $P$ and any line segment which meets the interior of $P$ at exactly once point.

Bocci and Franci considered the bipyramid of an $n$-gon. Specifically, let $Q_{n}$ be an $n$-gon in $\mathbb{R}^{2}$, with vertices $\{1, \ldots, n\}$, containing the origin and embedded in $\mathbb{R}^{3}$. We denote by $B_{n}$ the bipyramid over $Q_{n}$, i.e., the convex hull

$$
B_{n}=\operatorname{bipyr}\left(Q_{n}\right)=\operatorname{conv}\left(Q_{n},(0,0,1),(0,0,-1)\right) .
$$

For a simplicial complex $\Delta$ with vertices $\{1, \ldots, n\}$, we may identify a subset $\sigma \subseteq$ $\{1, \ldots, n\}$ with the $n$-tuple in $\{0,1\}^{n}$ and we adopt the convention that $x^{\sigma}=\prod_{i \in \sigma} x_{i}$. The Stanley-Reisner ideal of a simplicial complex $\Delta$ on vertices $\{1, \ldots, n\}$ is defined to be $I_{\Delta}=\left\langle x^{\sigma} \mid \sigma \notin \Delta\right\rangle$, i.e., it is generated by the non-faces of $\Delta$.

We view $B_{n}$ as a simplicial complex on the vertex set $\left\{x_{1}, \ldots, x_{n}, y, z\right\}$ where the $x_{i}$ 's correspond to the vertices of the $n$-gon, and $y$ and $z$ correspond to the end points of the line segment that meets the interior of the $n$-gon at one point. Because the bipyramid $B_{n}$ is a simplicial complex, we let $I_{n}=I_{B_{n}}$ be the Stanley-Reisner ideal associated to $B_{n}$. Bocci and Franci [2, Proposition 3.1] described the generators of $I_{n}$; in particular,

$$
\left.I_{n}=\langle y z\rangle+\left\langle x_{i} x_{j}\right| i \text { and } j \text { non-adjacent in } Q_{n}\right\rangle \text {. }
$$

Note that $I_{n}$ can be viewed as the edge ideal of some graph since all the generators are quadratic squarefree monomials. Using the results of this section, we have shown:

Theorem 6.10 ([2, Theorem 1.1]). Let $I_{n}$ be the Stanley-Reisner ideal of the n-bipyramid $B_{n}$. Then $\widehat{\alpha}\left(I_{n}\right)=\frac{n}{n-2}$ for all $n \geq 4$. 
Proof. The ideal $I_{n}$ is an ideal in the polynomial ring $R=k\left[x_{1}, \ldots, x_{n}, y, z\right]$. By (6.1) $I_{n}$ can be viewed as the edge ideal of the graph $G_{n}$ where $G_{n}=H \cup C_{n}^{c}$ consists of two disjoint components. In particular, $H$ is the graph of a single edge $\{y, z\}$ and $C_{n}^{c}$ is the complement of the $n$-cycle $C_{n}$. By Corollary 4.7 to compute $\widehat{\alpha}\left(I_{n}\right)$ it suffices to compute $\chi^{*}\left(G_{n}\right)=\max \left\{\chi^{*}(H), \chi^{*}\left(C_{n}^{c}\right)\right\}$. A graph consisting of a single edge is perfect, so $\chi^{*}(H)=2$. On the other hand,

$$
\chi^{*}\left(C_{n}^{c}\right)= \begin{cases}m & \text { if } n=2 m \\ m+\frac{1}{2} & \text { if } n=2 m+1\end{cases}
$$

So, if $n>3, \chi^{*}\left(G_{n}\right)=\chi^{*}\left(C_{n}^{c}\right)$.

Thus, if $n=2 m, \widehat{\alpha}\left(I_{n}\right)=\frac{m}{m-1}=\frac{n}{n-2}$. And if $n=2 m+1$, then

$$
\widehat{\alpha}\left(I_{n}\right)=\frac{m+\frac{1}{2}}{m-\frac{1}{2}}=\frac{n}{n-2} .
$$

In other words, $\widehat{\alpha}\left(I_{n}\right)=\frac{n}{n-2}$ for all $n \geq 4$.

We close this section with some comments about the Alexander dual.

Definition 6.11. Let $I=P_{1} \cap \cdots \cap P_{s}$ be a squarefree monomial ideal with $P_{i}=$ $\left\langle x_{j_{1}}, \ldots, x_{j_{s_{j}}}\right\rangle$ for $j=1, \ldots, s$. Then the Alexander dual of $I$, denoted $I^{\vee}$, is the monomial ideal $I^{\vee}=\left\langle x_{j_{1}} \cdots x_{j_{j}} \mid j=1, \ldots, s\right\rangle$.

In combinatorial commutative algebra, the Alexander dual of a monomial ideal $I$ is used quite frequently to deduce additional information about $I$. It is thus natural to ask if knowing $\widehat{\alpha}(I)$ of a squarefree monomial ideal allows us to deduce any information about $\widehat{\alpha}\left(I^{\vee}\right)$. As the next example shows, simply knowing $\widehat{\alpha}(I)$ gives no information on $\widehat{\alpha}\left(I^{\vee}\right)$.

Example 6.12. Let $s \geq 1$ be an integer, and let $G_{s}=K_{s, s}$ be the complete bipartite graph on the vertex set $V=\left\{x_{1}, \ldots, x_{s}\right\} \cup\left\{y_{1}, \ldots, y_{s}\right\}$. Now $\widehat{\alpha}\left(I\left(G_{s}\right)\right)=2$ by Theorem 6.7 for all $s \geq 1$. On the other hand, since $I\left(G_{s}\right)=\left\langle x_{1}, \ldots, x_{s}\right\rangle \cap\left\langle y_{1}, \ldots, y_{s}\right\rangle$, we have

$$
I\left(G_{s}\right)^{\vee}=\left\langle x_{1} \cdots x_{s}, y_{1} \cdots y_{s}\right\rangle .
$$

But the ideal $I\left(G_{s}\right)^{\vee}$ is a complete intersection so $\left(I\left(G_{s}\right)^{\vee}\right)^{(m)}=\left(I\left(G_{s}\right)^{\vee}\right)^{m}$ for all $m$. In particular, $\alpha\left(\left(I\left(G_{s}\right)^{\vee}\right)^{(m)}=\alpha\left(\left(I\left(G_{s}\right)^{\vee}\right)^{m}=s m\right.\right.$. So $\widehat{\alpha}\left(I\left(G_{s}\right)^{\vee}\right)=s$.

We see that if we only know that $\widehat{\alpha}(I)=2$, then $\widehat{\alpha}\left(I^{\vee}\right)$ can be any positive integer. We require further information about $I$ to deduce any information about $\widehat{\alpha}\left(I^{\vee}\right)$.

\section{Some applications to the ideal COntainment PROBlem}

As mentioned in the introduction, the renewed interest in the Waldschmidt constant grew out of the activity surrounding the containment problem for ideals of subschemes $X$ of $\mathbb{P}^{n}$, i.e., determine all positive integer pairs $(m, r)$ such that $I^{(m)} \subseteq I^{r}$ where $I=I(X)$. We apply our technique for computing $\widehat{\alpha}(I)$ to examine the containment problem for three families of monomial ideals: (1) a union of a small number (when compared to $n$ ) of general linear varieties, (2) the Stanley-Reisner ideal of a uniform matroid, and (3) a 
family of monomial ideals of mixed height. Note that for this section, we shall assume that $R=k\left[\mathbb{P}^{n}\right]=k\left[x_{0}, \ldots, x_{n}\right]$.

Before turning to our applications, we recall some relevant background. To study the containment problem, Bocci and Harbourne [3] introduce the resurgence of $I$, that is,

$$
\rho(I)=\sup \left\{\frac{m}{r} \mid I^{(m)} \not I^{r}\right\} .
$$

An asymptotic version of resurgence was later defined by Guardo, Harbourne, and Van Tuyl [16] as

$$
\rho_{a}(I)=\sup \left\{\frac{m}{r} \mid I^{(m t)} \nsubseteq I^{r t} \text { for all } t \gg 0 .\right\}
$$

These invariants are related to the Waldschmidt constant of $I$ as follows.

Lemma 7.1 ([16, Theorem 1.2]). Let $I \subseteq R=k\left[x_{0}, \ldots, x_{n}\right]$ be a homogeneous ideal. Then

(i) $1 \leq \alpha(I) / \widehat{\alpha}(I) \leq \rho_{a}(I) \leq \rho(I)$.

(ii) If $I$ is the ideal of a smooth subscheme of $\mathbb{P}^{n}$, then $\rho_{a}(I) \leq \omega(I) / \widehat{\alpha}(I)$ where $\omega(I)$ denotes the largest degree of a minimal generator.

7.1. Unions of general linear varieties. In [16, Theorem 1.5], the values of $\widehat{\alpha}(I)$ and $\rho(I)$ are established when $I$ is the ideal of certain linear subschemes of $\mathbb{P}^{n}$ in general position. The key idea is that when the number of linear varieties is small, we can assume that the defining ideal of $I$ is a monomial ideal. By using Theorem 3.2 to compute the Waldschmidt constant, we are able to recover and extend the original result.

Theorem 7.2. Let $X$ be the union of $s$ general linear subvarieties $L_{1}, \ldots, L_{s}$, each of dimension $t-1$. Assume st $\leq n+1$ and set $I=I(X)$. Then

$$
\widehat{\alpha}(I)= \begin{cases}1 & \text { if } 1 \leq s t<n+1 \\ \frac{n+1}{n+1-t} & \text { if } s t=n+1\end{cases}
$$

Additionally, if $s \geq 2$, then the resurgences are

$$
\rho(I)=\rho_{a}(I)=\frac{2 \cdot(s-1)}{s} .
$$

Furthermore,

$$
\begin{aligned}
& \frac{\alpha(I)}{\widehat{\alpha}(I)}=\rho_{a}(I)=\rho(I)=\frac{\omega(I)}{\widehat{\alpha}(I)}, \quad \text { if } n+1=s t \\
& \frac{\alpha(I)}{\widehat{\alpha}(I)}<\rho_{a}(I)=\rho(I)<\frac{\omega(I)}{\widehat{\alpha}(I)}, \quad \text { if } s t \leq n \text { and } s \geq 3 .
\end{aligned}
$$

Remark 7.3. If $s=1$, then the ideal $I$ of Theorem 7.2 is generated by variables, and so $\rho(I)=\rho_{a}(I)=1$. Thus, the assumption $s \geq 2$ is harmless. The case $t=1$ in Theorem 7.2 was first proved in [3], while the case $t=2$ is found in [16].

The final assertion of Theorem 7.2 gives examples where neither the lower bound nor the upper bound for the asymptotic resurgence in Lemma 7.1 are sharp.

As preparation, we note the following observation. 
Lemma 7.4. Let $0 \neq I \subset R$ be a monomial ideal, and let $y$ be a new variable. Consider the ideal $(I, y)$ in $S=R[y]$. Then

$$
\rho((I, y))=\rho(I) \quad \text { and } \quad \rho_{a}((I, y))=\rho_{a}(I) .
$$

Proof. First we show $\rho((I, y)) \geq \rho(I)$ and $\rho_{a}((I, y)) \geq_{a} \rho(I)$. To this end, assume $I^{(m t)} \nsubseteq$ $I^{r t}$ for some positive integers $m, r$ and $t$. Thus, there is a monomial $x^{a}=x_{0}^{a_{0}} \cdots x_{n}^{a_{n}} \in I^{(m t)}$ with $x^{a} \notin I^{r t}$. It follows that $x^{a} \in(I, y)^{(m t)}$, but $x^{a} \notin(I, y)^{r t}$, which implies both $\rho((I, y)) \geq \rho(I)$ and $\rho_{a}((I, y)) \geq \rho_{a}(I)$.

Second, we prove $\rho((I, y)) \leq \rho(I)$. Consider positive integers $m$ and $r$ with $\frac{m}{r}>\rho(I)$. It suffices to show $(I, y)^{(m)} \subseteq(I, y)^{r}$. To this end consider a minimal generator $x^{a} y^{b}$ of $(I, y)^{(m)}$. If $b \geq r$, then clearly $x^{a} y^{b} \in(I, y)^{r}$, and we are done. Otherwise, $b<r<m$, and $x^{a} y^{b} \in(I, y)^{(m)}$ gives $x^{a} \in I^{(m-b)}$. Now $\frac{m-b}{r-b} \geq \frac{m}{r}>\rho(I)$ implies $I^{(m-b)} \subseteq I^{r-b}$, and hence $x^{a} \in I^{r-b}$. It follows that $x^{a} y^{b}$ is in $(I, y)^{r}$, which shows $(I, y)^{(m)} \subseteq(I, y)^{r}$.

Similarly, one establishes $\rho_{a}((I, y)) \leq \rho_{a}(I)$

Proof of Theorem 7.2. Because the linear varieties $L_{i}$ are in general position, we may assume

$$
I\left(L_{i}\right)=\left(x_{0}, x_{1}, \ldots, \widehat{x}_{(i-1) t}, \ldots, \widehat{x}_{i t-1}, x_{i t}, \ldots, x_{n}\right),
$$

where the ${ }^{\wedge}$ denotes an omitted variable. In particular, $I(X)=\bigcap_{i=1}^{s} I\left(L_{i}\right)$ is a squarefree monomial ideal, so we can apply Theorem 3.2 to calculate $\widehat{\alpha}(I(X))$.

If $1 \leq s t<n+1$, we wish to minimize $x_{0}+x_{1}+\cdots+x_{n}$ subject to

$$
x_{0}+x_{1}+\cdots+\widehat{x}_{(i-1) t}+\cdots+\widehat{x}_{i t-1}+\cdots+x_{n} \geq 1 \text { for } i=1, \ldots, s .
$$

Since $s t<n+1$, the vector $\mathbf{y}^{T}=\left[\begin{array}{llll}0 & \cdots & 0 & 1\end{array}\right]$ is a feasible solution, so the minimum is at most 1 . On the other hand, because $x_{0}+\cdots+x_{n} \geq x_{t}+\cdots+x_{n} \geq 1$, the minimum solution is at least 1 . So $\widehat{\alpha}(I(X))=1$ in this situation.

If $s t=n+1$, then the matrix $A$ of Theorem 3.2 is an $s \times(n+1)$ matrix whose $i$-th row consists of $(i-1) t 1$ 's, followed by $t 0$ 's, followed by $n+1-i t 1$ 's. The vector $\mathbf{y}$ with

$$
\mathbf{y}^{T}=\underbrace{\left[\begin{array}{lll}
\frac{1}{n+1-t} & \cdots & \frac{1}{n+1-t}
\end{array}\right]}_{n+1}
$$

is a feasible solution to the linear program of Theorem 3.2 , and so $\widehat{\alpha}(I(X)) \leq \frac{n+1}{n+1-t}$. The associated dual linear program is as follows: maximize $y_{0}+\cdots+y_{n}$ such that $A^{T} \mathbf{y} \leq \mathbf{1}$. We claim that the $s$-tuple $\mathbf{y}$ with

$$
\mathbf{y}^{T}=\left[\begin{array}{lll}
\frac{t}{n+1-t} & \cdots & \frac{t}{n+1-t}
\end{array}\right]
$$

is a feasible solution. Indeed, observe that in each entry, $A^{T} \mathbf{y}$ is $(s-1)\left(\frac{t}{n+1-t}\right)=$ $\left(\frac{n+1-t}{t}\right)\left(\frac{t}{n+1-t}\right)=1$, and thus $\widehat{\alpha}(I(X)) \geq \frac{s t}{n+1-t}=\frac{n+1}{n+1-t}$. Combining inequalities gives us the desired result for the Waldschmidt constant.

For the remaining claims, we consider first the case where $n+1=s t$. Note that then $\alpha(I(X))=\omega(I(X))=2$. Moreover, $s \geq 2$ implies $t \leq \frac{n+1}{2}$. It follows that $2(t-1)=$ 
$\operatorname{dim} L_{i}+\operatorname{dim} L_{j}<n$, and thus $X$ is smooth. Hence, Lemma 7.1 gives

$$
\rho_{a}(I(X))=\frac{\alpha(I(X))}{\widehat{\alpha}(I(X))}=\frac{2}{(n+1) /(n+1-t)}=\frac{2 \cdot(s-1)}{s} \leq \rho(I(X)) .
$$

Thus, in order to determine $\rho(I(X))$ it suffices to show: If $m$ and $r$ are positive integers with $\frac{m}{r}>\frac{2 \cdot(s-1)}{s}$, then $\left.I(X)\right)^{(m)} \subset I(X)^{r}$. To this end we adapt the argument employed in the proof of [16, Theorem 1.5]. Consider the ring homomorphism

$$
\varphi: R \rightarrow S=k\left[y_{0}, \ldots, y_{s-1}\right] \text {, defined by } x_{i} \mapsto y_{j} \text { if } j t \leq i<(j+1) t .
$$

Note that, for each $i \in[s]=\{1, \ldots, s\}$, the ideal of $S$ generated by $\varphi\left(I\left(L_{i}\right)\right)$ is $P_{i}=$ $\left(y_{0}, \ldots, \widehat{y}_{i-1}, \ldots, y_{s-1}\right)$. Thus, $J=P_{1} \cap \cdots \cap P_{s}$ is the ideal of the $s$ coordinate points in $\mathbb{P}^{s-1}$.

Consider a monomial $x^{a}=x_{0}^{a_{0}} \cdots x_{n}^{a_{n}} \in R$. Then $x^{a}$ is in $I^{(m)}=\bigcap_{i=1}^{s} I\left(L_{i}\right)^{m}$ if and only if $\operatorname{deg}\left(x^{a}\right)-\left(a_{(i-1) t}+a_{(i-1) t+1}+\cdots+a_{i t-1}\right) \geq m$ for each $i \in[s]$. Furthermore, a monomial $y^{b}=y_{0}^{b_{0}} \cdots y_{s-1}^{b_{s-1}} \in S$ is in $J^{(m)}=\bigcap_{i=1}^{s} P_{i}^{m}$ if and only if $\operatorname{deg}\left(y^{b}\right)-b_{i-1} \geq m$ for every $i \in[s]$. It follows that

$$
x^{a} \in I^{(m)} \text { if and only if } \varphi\left(x^{a}\right) \in J^{(m)} .
$$

Consider now any monomial $x^{a}$ in $I^{(m)}$. The equivalence (7.1) gives $\varphi\left(x^{a}\right) \in J^{(m)}$. Since $\rho(J)=\frac{2 \cdot(s-1)}{s}$ by [3, Theorem 2.4.3], our assumption $\frac{m}{r}>\frac{2 \cdot(s-1)}{s}$ yields $J^{(m)} \subseteq J^{r}$. Hence, we can write $\varphi\left(x^{a}\right)=\pi_{1} \cdots \pi_{r}$, where each $\pi_{j}$ is a monomial in $J$. Equivalence (7.1) implies now that there are monomials $\mu_{1}, \ldots, \mu_{r} \in I$ such that $\varphi\left(\mu_{j}\right)=\pi_{j}$ for each $j$ and $x^{a}=\mu_{1} \cdots \mu_{r}$. It follows that $x^{a} \in I^{r}$, and hence $I^{(m)} \subset I^{r}$, as desired.

Finally, assume $n \geq s t$. Then $I(X)$ is the sum of $n+1-s t$ variables and the extension ideal $I(Y) R$ of the ideal of the union $Y$ of $s$ general $(t-1)$-dimensional linear subspaces in $\mathbb{P}^{s t-1}$. Thus, Lemma 7.4 yields $\rho(I(X))=\rho(I(Y))$ and $\rho_{a}(I(X))=\rho_{a}(I(Y))$, and hence $\rho(I(X))=\rho_{a}(I(X))=\frac{2 \cdot(s-1)}{s}$. However, $\frac{\alpha(I(X))}{\widehat{\alpha}(I(X))}=\frac{1}{1}=1$ and $\frac{\omega(I(X))}{\widehat{\alpha}(I(X))}=\frac{2}{1}=2$.

7.2. Stanley-Reisner ideals of uniform matroids. We use our methods to determine the Waldschmidt constant of the Stanley-Reisner ideal $I_{n+1, c}$ of a uniform matroid $\Delta$ on $n+1$ vertices whose facets are all the cardinality $n+1-c$ subsets of the vertex set. These ideals were also recently studied by Geramita, Harbourne, Migliore, and Nagel [15] and Lampa-Baczyńska and Malara [21. The ideal $I_{n+1, c}$ is generated by all squarefree monomials of degree $n+2-c$ in $R$. Equivalently,

$$
I_{n+1, c}=\bigcap_{0 \leq i_{1}<i_{2}<\cdots<i_{c} \leq n}\left(x_{i_{1}}, x_{i_{2}}, \ldots, x_{i_{c}}\right) .
$$

Theorem 7.5. The Stanley-Reisner ideal of a $(n-c)$-dimensional uniform matroid on $n+1$ vertices has Waldschmidt constant

$$
\widehat{\alpha}\left(I_{n+1, c}\right)=\frac{n+1}{c} .
$$

Proof. This follows by [3, Lemma 2.4.1, Lemma 2.4.2 and the proof of Theorem 2.4.3]. However, we wish to provide a direct argument here. 
THE WALDSCHMIDT CONSTANT FOR SQUAREFREE MONOMIAL IDEALS

We use Theorem 3.2. By Equation (7.2), each left-hand side of the $\left(\begin{array}{c}s+1 \\ c\end{array}\right)$ inequalities in $A \mathbf{y} \geq \mathbf{1}$ is a sum of $c$ distinct variables in $\left\{y_{1}, \ldots, y_{n+1}\right\}$. Thus,

$$
y_{1}=\cdots=y_{n+1}=\frac{1}{c}
$$

is a feasible solution, which gives $\widehat{\alpha}\left(I_{n+1, c}\right) \leq \frac{n+1}{c}$.

Now observe that each $y_{i}$ appears in $\left(\begin{array}{c}n \\ c-1\end{array}\right)$ inequalities of $A \mathbf{y} \geq \mathbf{1}$. Hence, summing over all these inequalities we get

$$
\left(\begin{array}{c}
n \\
c-1
\end{array}\right)\left[y_{1}+\cdots+y_{n+1}\right] \geq\left(\begin{array}{c}
n+1 \\
c
\end{array}\right),
$$

which yields $\widehat{\alpha}\left(I_{n+1, c}\right) \geq\left(\begin{array}{c}n+1 \\ c\end{array}\right) /\left(\begin{array}{c}n \\ c-1\end{array}\right)=\frac{n+1}{c}$, completing the argument.

Remark 7.6. The ideal $I_{n+1, c}$ give an example of a family of squarefree monomial ideals that achieves the lower bound of Theorem 5.3. Indeed, big-height $\left(I_{n+1, c}\right)=c$ and $\alpha\left(I_{n+1, c}\right)=n+2-c$, so

$$
\widehat{\alpha}\left(I_{n+1, c}\right)=\frac{n+1}{c}=\frac{\alpha(I)+c-1}{c} .
$$

7.3. A binomial-like theorem for symbolic powers of monomial ideals. In this subsection we introduce some results which will be useful in Subsection 7.4. They are also of independent interest.

Lemma 7.7. Let $I_{1}$ and $I_{2}$ be squarefree monomial ideals whose generators are expressed in terms of disjoint sets of variables $x_{1}, \ldots, x_{n}$ and $y_{1}, \ldots, y_{m}$, respectively. Let $\operatorname{Ass}\left(I_{1}\right)=$ $\left\{P_{1}, \ldots, P_{a}\right\}$ and $\operatorname{Ass}\left(I_{2}\right)=\left\{Q_{1}, \ldots, Q_{b}\right\}$. Then

$$
I_{1}^{(s)} I_{2}^{(t)}=\bigcap_{i, j} P_{i}^{s} Q_{j}^{t}
$$

Proof. All of these ideals are monomial. Since $P_{i}^{s}$ and $Q_{j}^{t}$ are ideals written in terms of distinct sets of variables, $P_{i}^{s} \cap Q_{j}^{t}=P_{i}^{s} Q_{j}^{t}$ and by the same reasoning $I_{1}^{(s)} \cap I_{2}^{(t)}=I_{1}^{(s)} I_{2}^{(t)}$. Thus

$$
\bigcap_{i, j} P_{i}^{s} Q_{j}^{t}=\bigcap_{i, j}\left(P_{i}^{s} \cap Q_{j}^{t}\right)=\left(\bigcap_{i} P_{i}^{s}\right) \cap\left(\bigcap_{j} Q_{j}^{t}\right)=I_{1}^{(s)} \cap I_{2}^{(t)}=I_{1}^{(s)} I_{2}^{(t)} .
$$

From here, we deduce the symbolic binomial theorem.

Theorem 7.8. Let $I_{1}$ and $I_{2}$ be squarefree monomial ideals in variables $x_{1}, \ldots, x_{n}$ and $y_{1}, \ldots, y_{m}$, respectively. Set $I=I_{1}+I_{2}$. Then

$$
I^{(m)}=\sum_{j=0}^{m} I_{1}^{(m-j)} I_{2}^{(j)}
$$


Proof. Using the notation of Lemma 7.7, the associated primes of $I$ are of the form $P_{i}+Q_{j}$ for all $i, j$. Thus,

$$
\begin{aligned}
I^{(m)} & =\bigcap_{i, j}\left(P_{i}+Q_{j}\right)^{m}=\bigcap_{i, j}\left(P_{i}^{m}+P_{i}^{m-1} Q_{j}+\cdots+P_{i} Q_{j}^{m-1}+Q_{j}^{m}\right) \\
& =\bigcap_{i} P_{i}^{m}+\bigcap_{i, j} P_{i}^{m-1} Q_{j}+\cdots+\bigcap_{i, j} P_{i} Q_{j}^{m-1}+\bigcap_{j} Q_{j}^{m} \\
& =I_{1}^{(m)}+I_{1}^{(m-1)} I_{2}+\cdots+I_{1} I_{2}^{(m-1)}+I_{2}^{(m)},
\end{aligned}
$$

where the third equality follows by use of the modular law for monomial ideals (i.e. $L \cap(J+K)=L \cap J+L \cap K$ for monomial ideals $J, K, L)$ and the last equality follows from Lemma 7.7 .

We give to applications for this theorem.

Corollary 7.9. If $G_{1}$ and $G_{2}$ are disjoint graphs, then

$$
I\left(G_{1} \cup G_{2}\right)^{(m)}=\sum_{j=0}^{m} I\left(G_{1}\right)^{(m-j)} I\left(G_{2}\right)^{(j)} .
$$

As a second application, we are able to give a new proof for Corollary 4.7. Unlike our first proof, which is combinatorial, the methods of this new proof are entirely algebraic.

Corollary 7.10. Suppose that $I$ and $J$ are two squarefree monomial ideals of the ring $R=k\left[x_{1}, \ldots, x_{n}, y_{1}, \ldots, y_{n}\right]$. Furthermore, suppose that $I$ is generated by monomials only in the $x_{i}$ 's and $J$ is generated by monomials only in the $y_{j}$ 's. Then

$$
\widehat{\alpha}(I+J)=\min \{\widehat{\alpha}(I), \widehat{\alpha}(J)\} .
$$

Proof. Since $I^{(n)} \subseteq(I+J)^{(n)}$, we deduce that $\alpha\left(I^{(n)}\right) \geq \alpha\left((I+J)^{(n)}\right)$ for all $n \geq 0$. Thus we also have the inequality $\widehat{\alpha}(I) \geq \widehat{\alpha}(I+J)$ and similarly we obtain $\widehat{\alpha}(J) \geq \widehat{\alpha}(I+J)$. For the reverse inequality, from Theorem 7.8 , we deduce that

$\alpha\left((I+J)^{(n)}\right)=\min _{0 \leq m \leq n} \alpha\left(I^{(m)} J^{(n-m)}\right) \geq \min _{0 \leq m \leq n}(m \widehat{\alpha}(I)+(n-m) \widehat{\alpha}(J)) \geq n \cdot \min (\widehat{\alpha}(I), \widehat{\alpha}(J))$, which allows to conclude $\widehat{\alpha}(I+J) \geq \min (\widehat{\alpha}(I), \widehat{\alpha}(J))$.

7.4. Monomial ideals of mixed height. For many families of ideals $I$ for which we know $\widehat{\alpha}(I)$ (or $\rho_{a}(I)$ and $\left.\rho(I)\right)$, the ideal $I$ is unmixed, i.e., all of the associated primes of $I$ have the same height. In this final part of the paper, we present some initial results on the problem of computing these invariants for ideals of mixed height.

In particular, as a case study we focus on the scheme $Z \subset \mathbb{P}^{n}(n \geq 2)$ defined by $n$ general $(n-2)$-planes in an $(n-1)$-plane and one point out of the plane (a "monomial star"). This scheme is similar to the type of varieties studied by Fatabbi, Harbourne, and Lorenzini [12]. Specifically, we wish to consider the family of ideals

$$
\begin{aligned}
I_{Z} & =\left(x_{0} x_{n}, x_{1} x_{n}, \ldots, x_{n-1} x_{n}, x_{0} x_{1} \cdots x_{n-1}\right) \\
& =\left(x_{0} \cdots x_{n-1}, x_{n}\right) \cap\left(x_{0}, \ldots, x_{n-1}\right)=P_{0} \cap P_{1} \cap \cdots \cap P_{n-1} \cap P_{n},
\end{aligned}
$$

where for $0 \leq i \leq n-1 P_{i}=\left(x_{i}, x_{n}\right)$ for $i=1, \ldots, n$ and $P_{n}=\left(x_{0}, \ldots, x_{n-1}\right)$. 
We wish to obtain information about containments of the form $I_{Z}^{(m)} \subseteq I_{Z}^{r}$. Theorem 3.2 enables us to easily compute the Waldschmidt constant for these ideals, and consequently, a lower bound on the (asymptotic) resurgence.

Lemma 7.11. With $Z \subseteq \mathbb{P}^{n}$ defined as above, we have

$$
\widehat{\alpha}\left(I_{Z}\right)=\frac{2 n-1}{n} .
$$

Proof. The second statement follows from Theorem 7.1. We know that $\widehat{\alpha}\left(I_{Z}\right)$ is the value of the LP obtained by minimizing $\mathbf{b}^{T} \mathbf{y}=\mathbf{1}^{T} \mathbf{y}$ subject to $A \mathbf{y} \geq \mathbf{1}=\mathbf{c}$ and $\mathbf{y} \geq \mathbf{0}$ where

$$
A=\left[\begin{array}{ccccccc}
1 & 0 & 0 & \cdots & 0 & 0 & 1 \\
0 & 1 & 0 & \cdots & 0 & 0 & 1 \\
\vdots & \vdots & \vdots & \vdots & \vdots & \vdots & \vdots \\
0 & 0 & 0 & \cdots & 0 & 1 & 1 \\
1 & 1 & 1 & \cdots & 1 & 1 & 0
\end{array}\right]
$$

A feasible solution is

$$
\mathbf{y}^{T}=\left[\begin{array}{lllll}
\frac{1}{n} & \frac{1}{n} & \cdots & \frac{1}{n} & \frac{n-1}{n}
\end{array}\right] .
$$

To see that this solution is optimal, note that the matrix $A$ is symmetric and $\mathbf{b}=\mathbf{c}=\mathbf{1}$, and so this solution is also a feasible solution to the dual linear program. Therefore, $\widehat{\alpha}\left(I_{Z}\right)=\mathbf{1}^{T} \mathbf{y}=(2 n-1) / n$.

We will compute the resurgence for the ideals $I_{Z}$.

Lemma 7.12. Let $0 \neq I \subset R$ be a squarefree monomial ideal such that $I^{(m)}=I^{m}$ for all $m \geq 0$. Let $y$ be a new variable and consider the ideal $(I, y)$ in $S=R[y]$. Then

$$
(I, y)^{(m)}=(I, y)^{m} \text { for all } \geq 0 .
$$

Proof. By Theorem 7.8, we have

$$
(I, y)^{(m)}=\sum_{m_{1}+m_{2}=m} I^{\left(m_{1}\right)}(y)^{\left(m_{2}\right)}=\sum_{m_{1}+m_{2}=m} I^{m_{1}} y^{m_{2}}=(I, y)^{m} .
$$

We now collect together a number of results that we will require regarding the symbolic and ordinary powers of $I_{Z}$.

Lemma 7.13. With $Z \subseteq \mathbb{P}^{n}$ defined as above, we have

(i) $I_{Z}^{(m)}=\left(x_{0} x_{1} \cdots x_{n-1}, x_{n}\right)^{m} \cap\left(x_{0}, x_{1}, \ldots, x_{n-1}\right)^{m}$.

(ii) $I_{Z}^{(m)}=\sum_{i=0}^{\left\lfloor\frac{(n-1) m}{n}\right\rfloor} x_{n}^{i}\left(x_{0} \cdots x_{n-1}\right)^{m-i}+\sum_{i=\left\lfloor\frac{(n-1) m}{n}\right\rfloor+1}^{m} x_{n}^{i}\left(x_{0} \cdots x_{n-1}\right)^{m-i}\left(x_{0}, \ldots, x_{n-1}\right)^{n i-(n-1) m}$

(iii) $I^{s}=\sum_{i=0}^{s} x_{n}^{i}\left(x_{0} \cdots x_{n-1}\right)^{s-i}\left(x_{0}, \ldots, x_{n-1}\right)^{i}$.

Proof. For $(i)$, by Lemma 7.12 we have

$$
\begin{aligned}
I_{Z}^{(m)} & =\left(x_{0}, x_{n}\right)^{m} \cap\left(x_{1}, x_{n}\right)^{m} \cap \ldots \cap\left(x_{n-1}, x_{n}\right)^{m} \cap\left(x_{0}, \ldots, x_{n-1}\right)^{m} \\
& =\left(x_{0} \cdots x_{n-1}, x_{n}\right)^{(m)} \cap\left(x_{0}, \ldots, x_{n-1}\right)^{m}=\left(x_{0} \cdots x_{n-1}, x_{n}\right)^{m} \cap\left(x_{0}, \ldots, x_{n-1}\right)^{m} .
\end{aligned}
$$


For $(i i)$ we have

$$
\begin{aligned}
I^{(m)} & =\left(x_{0} \cdots x_{n-1}, x_{n}\right)^{m} \cap\left(x_{0}, \ldots, x_{n-1}\right)^{m}=\sum_{i=0}^{m}\left(x_{n}^{i}\left(x_{0} \cdots x_{n-1}\right)^{m-i} \cap\left(x_{0}, \ldots, x_{n-1}\right)^{m}\right) \\
& =\sum_{i=0}^{\lfloor(n-1) m / n\rfloor} x_{n}^{i}\left(x_{0} \cdots x_{n-1}\right)^{m-i}+\sum_{i=\lfloor(n-1) m / n\rfloor+1}^{m} x_{n}^{i}\left(x_{0} \cdots x_{n-1}\right)^{m-i}\left(x_{0}, \ldots, x_{n-1}\right)^{m-n(m-i)} \\
& =\sum_{i=0}^{\lfloor(n-1) m / n\rfloor} x_{n}^{i}\left(x_{0} \cdots x_{n-1}\right)^{m-i}+\sum_{i=\lfloor(n-1) m / n\rfloor+1}^{m} x_{n}^{i}\left(x_{0} \cdots x_{n-1}\right)^{m-i}\left(x_{0}, \ldots, x_{n-1}\right)^{n i-(n-1) m} .
\end{aligned}
$$

Finally, for $($ iii $)$, we have

$$
\begin{aligned}
I^{s} & =\left(x_{0} \cdots x_{n-1}, x_{0} x_{n}, x_{1} x_{n}, \ldots, x_{n-1} x_{n}\right)^{s} \\
& =\sum_{i=0}^{s}\left(x_{0} \cdots x_{n-1}\right)^{s-i}\left(x_{n}\left(x_{0}, \ldots, x_{n-1}\right)\right)^{i}=\sum_{i=0}^{s} x_{n}^{i}\left(x_{0} \cdots x_{n-1}\right)^{s-i}\left(x_{0}, \ldots, x_{n-1}\right)^{i} .
\end{aligned}
$$

Theorem 7.14. With $Z \subseteq \mathbb{P}^{n}$ defined as above, let $I=I_{Z}$. Then

(i) if $m, s$ are positive integers with $\frac{m}{s} \geq \frac{n^{2}}{n^{2}-n+1}$, then $I^{(m)} \subseteq I^{s}$

(ii) $I^{\left(n^{2} k\right)} \nsubseteq I^{\left(n^{2}-n+1\right) k+1}$, for all integers $k \geq 0$.

Consequently, $\rho\left(I_{Z}\right)=\rho_{a}\left(I_{Z}\right)=\frac{n^{2}}{n^{2}-n+1}$.

Proof. For $(i)$ it suffices to prove that each term in the decomposition of $I^{(m)}$ of Lemma 7.13 is contained in $I^{s}$. By Lemma 7.13 we have to analyze the following cases:

Case 1: if $0 \leq i \leq\left\lfloor\frac{(n-1) m}{n}\right\rfloor$, we will prove that

$$
x_{n}^{i}\left(x_{0} \cdots x_{n-1}\right)^{m-i} \in x_{n}^{i}\left(x_{0} \cdots x_{n-1}\right)^{s-i}\left(x_{0}, \ldots, x_{n-1}\right)^{i} .
$$

This is equivalent to $\left(x_{0} \cdots x_{n-1}\right)^{m-s} \in\left(x_{0}, \ldots, x_{n-1}\right)^{i}$, which is further equivalent to $n(m-s) \geq i$. To prove the latter inequality holds, it is sufficient to show that $n(m-s) \geq$ $\frac{(n-1) m}{n}$. Elementary manipulations show that this inequality is equivalent to the hypothesis $\frac{m}{s} \geq \frac{n^{2}}{n^{2}-n+1}$.

Case 2: if $\left\lfloor\frac{(n-1) m}{n}\right\rfloor+1 \leq i \leq \min \{m, s\}$, we will prove that

$$
x_{n}^{i}\left(x_{0} \cdots x_{n-1}\right)^{m-i}\left(x_{0}, \ldots, x_{n-1}\right)^{n i-(n-1) m} \subseteq x_{n}^{i}\left(x_{0} \cdots x_{n-1}\right)^{s-i}\left(x_{0}, \ldots, x_{n-1}\right)^{i} .
$$

This is equivalent to $\left(x_{0} \cdots x_{n-1}\right)^{m-s} \in\left(x_{0}, \ldots, x_{n-1}\right)^{(n-1)(m-i)}$. The latter is equivalent to $n(m-s) \geq(n-1)(m-i)$, or $m+(n-1) i \geq n s$, which holds true since $m+(n-1) i>$ $m+(n-1) \cdot \frac{(n-1) m}{n}=m \cdot \frac{n^{2}-n+1}{n} \geq n s$. The last inequality uses the hypothesis $\frac{m}{s} \geq \frac{n^{2}}{n^{2}-n+1}$. Case 3: if $\min \{m, s\}<i \leq m$, we will prove that

$$
x_{n}^{i}\left(x_{0} \cdots x_{n-1}\right)^{m-i}\left(x_{0}, \ldots, x_{n-1}\right)^{n i-(n-1) m} \subseteq x_{n}^{s}\left(x_{0}, \ldots, x_{n-1}\right)^{s} .
$$

For that, it suffices to prove that $n(m-i) \geq s+(n-1) m-n i$, which is equivalent to $m \geq s$. Either this inequality is satisfied or else this case is vacuous. 
For $(i i)$ the following notation will be used in the proof. For a monomial $f, \operatorname{deg}_{1} f$ denotes the total degree of the monomial part involving the variables $x_{0}, \ldots, x_{n-1}$, while $\operatorname{deg}_{2} f$ denotes exponent of $x_{n}$ in $f$. For two monomials $f$ and $g$, if $f$ is a multiple of $g$, then clearly $\operatorname{deg}_{1} f \geq \operatorname{deg}_{1} g$, and $\operatorname{deg}_{2} f \geq \operatorname{deg}_{2} g$.

Consider the monomial $f=\left(x_{0} \cdots x_{n-1}\right)^{n k} x_{n}^{(n-1) n k}$ in $I^{\left(n^{2} k\right)}$. Now assume that $f \in I^{s}$, where $s=\left(n^{2}-n+1\right) k+1$. Then there exists a minimal generator $g$ in $I^{s}$ such that $g \mid f$. Such a monomial $g$ has the form $\left(x_{0} \cdots x_{n-1}\right)^{s-\alpha} x_{0}^{t_{0}} \cdots x_{n-1}^{t_{n-1}} x_{n}^{\alpha}$, where $t_{0}, \ldots, t_{n-1}$ are nonnegative integers such that $t_{0}+\cdots+t_{n-1}=\alpha$. We have $\operatorname{deg}_{2} f=(n-1) n k \geq \operatorname{deg}_{2} g=\alpha$. Furthermore, $\operatorname{deg}_{1} f=n^{2} k \geq \operatorname{deg}_{1} g=n(s-\alpha)+\alpha$. In particular, this implies

$$
\alpha \geq \frac{n(s-n k)}{n-1}>\frac{n\left(n^{2}-2 n+1\right) k}{n-1}=n(n-1) k,
$$

which is a contradiction, so $(i i)$ follows.

The non-containment relations in part $(i i)$ yield

$$
\rho(I) \geq \sup \left\{\frac{n^{2} k}{\left(n^{2}-n+1\right) k+1} \mid k>0\right\}=\lim _{k \rightarrow \infty} \frac{n^{2} k}{\left(n^{2}-n+1\right) k+1}=\frac{n^{2}}{n^{2}-n+1},
$$

while part $(i)$ shows that the opposite inequality holds. We conclude that $\rho\left(I_{Z}\right)=\frac{n^{2}}{n^{2}-n+1}$.

As for the asymptotic resurgence, since $\rho_{a}(I) \leq \rho(I)$, we have $\rho_{a}(I) \leq \frac{n^{2}}{n^{2}-n+1}$. From part $(i i)$, since $I^{\left(\left(n^{2}-n+1\right) k+1\right) t} \subseteq I^{\left(\left(n^{2}-n+1\right) k t+1\right.}$ for $t \geq 1$, we deduce

$$
I^{\left(n^{2} k t\right)} \nsubseteq I^{\left(\left(n^{2}-n+1\right) k+1\right) t} \text { for } t \geq 1 \text {. }
$$

It follows that

$$
\rho_{a}(I) \geq \lim _{k \rightarrow \infty} \frac{n^{2} k}{\left(n^{2}-n+1\right) k+1}=\frac{n^{2}}{n^{2}-n+1},
$$

allowing us to conclude that $\rho_{a}\left(I_{Z}\right)=\frac{n^{2}}{n^{2}-n+1}$.

Remark 7.15. Similar to Remark 7.3, we have the following inequalities

$$
\begin{gathered}
\frac{\alpha\left(I_{Z}\right)}{\widehat{\alpha}\left(I_{Z}\right)}=\rho_{a}\left(I_{Z}\right)=\rho\left(I_{Z}\right)=\frac{\omega\left(I_{Z}\right)}{\widehat{\alpha}\left(I_{Z}\right)}=\frac{4}{3}, \text { if } n=2 \\
\frac{2 n}{2 n-1}=\frac{\alpha\left(I_{Z}\right)}{\widehat{\alpha}\left(I_{Z}\right)}<\rho_{a}\left(I_{Z}\right)=\rho\left(I_{Z}\right)<\frac{\omega\left(I_{Z}\right)}{\widehat{\alpha}\left(I_{Z}\right)}=\frac{n^{2}}{2 n-1}, \text { if } n>2
\end{gathered}
$$

for the family of ideals $I_{Z}$. The case $n=2$ corresponds to $Z$ being a set of 3 points in $\mathbb{P}^{2}$.

\section{REFERENCES}

[1] M. Baczyńska, M. Dumnicki, A. Habura, G. Malara, P. Pokora, T. Szemberg, J. Szpond, H. TutajGasińska, Points fattening on $\mathbb{P}^{1} \times \mathbb{P}^{1}$ and symbolic powers of bi-homogeneous ideals. J. Pure Appl. Algebra 218 (2014), no. 8, 1555-1562.

[2] C. Bocci, B. Franci, Waldschmidt constants for Stanley-Reisner ideals of a class of simplicial complexes. J. Algebra Appl. 15 (2016), no. 6, 1650137 (13 pages)

(2015) Preprint. arXiv: 1504.04201v1

[3] C. Bocci, B. Harbourne, Comparing powers and symbolic powers of ideals. J. Algebraic Geom. 19 (2010), no. 3, 399-417. 
[4] C. Bocci and B. Harbourne, The resurgence of ideals of points and the containment problem. Proc. Amer. Math. Soc. 138 (2010), no. 4, 1175-1190.

[5] G. V. Chudnovsky, Singular points on complex hypersurfaces and multidimensional Schwarz lemma. Seminar on Number Theory, Paris 1979-80, pp. 29-69, Progr. Math., 12, Birkhäuser, Boston, Mass., 1981.

[6] S. M. Cooper, R. J. D. Embree, H. T. Hà, A. H. Hoefel, Symbolic Powers of Monomial Ideals. (2013) To appear in Proc. Edinb. Math. Soc. (2). arXiv:1309.5082v3

[7] M. Dumnicki, Containments of symbolic powers of ideals of generic points in $\mathbb{P}^{3}$. Proc. Amer. Math. Soc. 143 (2015), no. 2, 513-530.

[8] M. Dumnicki, B. Harbourne, U. Nagel, A. Seceleanu, T. Szemberg, H. Tutaj-Gasińska, Resurgences for ideals of special point configurations in $\mathbb{P}^{N}$ coming from hyperplane arrangements. J. Algebra 443 (2015), 383-394.

[9] M. Dumnicki, B. Harbourne, T. Szemberg, H. Tutaj-Gasińska, Linear subspaces, symbolic powers and Nagata type conjectures. Adv. Math. 252 (2014), 471-491.

[10] L. Ein, R. Lazarsfeld and K. Smith. Uniform Behavior of Symbolic Powers of Ideals. Invent. Math. 144 (2001), no. 2, 241-252.

[11] H. Esnault and E. Viehweg. Sur une minoration du degré d'hypersurfaces s'annulant en certains points. Math. Ann. 263 (1983), no. 1, 75-86.

[12] G. Fatabbi, B. Harbourne, A. Lorenzini, Inductively computable unions of fat linear subspaces. (2015) To appear in J. Pure Appl. Algebra.

[13] H. Fekete, Über die Verteilung der Wurzeln bei gewissen algebraischen Gleichungen mit ganzzahligen Koeffizienten. Math. Z. 17 (1923), no. 1, 228-249.

[14] B. Franci, Costanti di Waldschmidt di Ideali di Stanley-Reisner di Bipiramidi. Tesi di Laurea, Università di Siena 2014.

[15] A. V. Geramita, B. Harbourne, J. Migliore, U. Nagel, Matroid configurations and symbolic powers of their ideals. (2015) To appear in Trans. Amer. Math. Soc. arXiv:1507.00380v1

[16] E. Guardo, B. Harbourne, A. Van Tuyl, Asymptotic resurgences for ideals of positive dimensional subschemes of projective space. Adv. Math. 246 (2013) 114-127.

[17] E. Guardo, B. Harbourne, A. Van Tuyl, Symbolic powers versus regular powers of ideals of general points in $\mathbb{P}^{1} \times \mathbb{P}^{1}$. Canad. J. Math. 65 (2013), no. 4, 823-842.

[18] B. Harbourne, C. Huneke, Are symbolic powers highly evolved? J. Ramanujan Math. Soc. 28A (2013), 247-266.

[19] B. Harbourne, J. Roé, Computing multi-point Seshadri constants on $\mathbb{P}^{2}$. Bull. Belg. Math. Soc. Simon Stevin 16 (2009), no. 5, 887-906.

[20] M. Hochster and C. Huneke. Comparison of symbolic and ordinary powers of ideals. Invent. Math. 147 (2002), no. 2, 349-369.

[21] M. Lampa-Baczyńska, G. Malara, On the containment hierarchy for simplicial ideals. J. Pure Appl. Algebra 219 (2015), no. 12, 5402-5412.

[22] R. Lazarsfeld, Positivity in algebraic geometry. I. Springer-Verlag, Berlin, 2004.

[23] E. Scheinerman, D. Ullman, Fractional graph theory. A rational approach to the theory of graphs. John Wiley \& Sons, Inc., New York, 1997.

[24] K. Schwede, A canonical linear system associated to adjoint divisors in characteristic $p>0$. J. Reine Angew. Math. 696 (2014), 69-87.

[25] A. Simis, W. Vasconcelos, R. Villareal, On the ideal theory of graphs. J. Algebra 167 (1994), no. 2, $389-416$.

[26] H. Skoda, Estimations $L^{2}$ pour l'opérateur $\widehat{\partial}$ et applications arithmétiques. In: Séminaire P. Lelong (Analyse), 1975/76, Lecture Notes Math. 578, Springer, 1977, 314-323.

[27] A. Van Tuyl, A beginner's guide to edge and cover ideals. In: Monomial ideals, computations and applications, Lecture Notes Math. 2083, Springer, 2013, 63-94.

[28] M. Waldschmidt, Propriétés arithmétiques de fonctions de plusieurs variables. II. In Séminaire P. Lelong (Analyse), 1975/76, Lecture Notes Math. 578, Springer, 1977, 108-135. 
[29] M. Waldschmidt. Nombres transcendants et groupes algébriques, Astérisque 69/70, Socéte Mathématiqué de France, 1979.

Department of Information Engineering and Mathematics, University of Siena, Via Roma, 56 Siena, Italy

E-mail address: cristiano.bocci@unisi.it

Department of Mathematics, North Dakota State University, NDSU Dept \#2750, PO Box 6050, FARGO, ND 58108-6050, USA

E-mail address: susan.marie.cooper@ndsu.edu

Dipartimento di Matematica e Informatica, Viale A. Doria, 6, 95100 - Catania, Italy

E-mail address: guardo@dmi.unict.it

Department of Mathematics, University of Nebraska, Lincoln, NE 68588-0130, USA

E-mail address: bharbourne1@unl.edu

Department of Mathematics, Statistics, and Computer Science, Dordt College, Sioux Center, IA 51250, USA

E-mail address: mike.janssen@dordt.edu

Department of Mathematics, University of Kentucky, 715 Patterson Office Tower, LEXINGTON, KY 40506-0027, USA

E-mail address: uwe.nagel@uky.edu

Department of Mathematics, University of Nebraska, Lincoln, NE 68588-0130, USA

E-mail address: aseceleanu@unl.edu

Department of Mathematics and Statistics, McMaster University, Hamilton, ON, L8S 4L8, CANADA

E-mail address: vantuyl@math.mcmaster.ca

Department of Mathematics, University of Nebraska, Lincoln, NE 68588-0130, USA

E-mail address: tvu@unl.edu 